\title{
Zur Multimodalität von Unterstützungsinteraktion
}

\begin{abstract}
Die Fallanalyse rekonstruiert aus multimodaler Perspektive eine Unterstützungsinteraktion im Unterricht. Die Unterstützung wird dabei als gemeinsame Herstellung des daran beteiligten Schülers und Lehrers konzeptualisiert. Es werden detailliert die vom Schüler produzierten Hinweise auf seine „Hilfsbedürftigkeit“ und die vom Lehrer eingesetzten Ressourcen bei seiner Hilfeleistung konstitutionsanalytisch rekonstruiert. In der falltranszendierenden Theoretisierung wird mit Bezug auf das gesprächsrhetorische Konzept „Unterstützen“ die Spezifik interaktiver Hilfeleistungen im Unterricht als konstitutive Anforderung an das professionelle Handeln von Lehrer/innen reflektiert.

This single case analysis reconstructs supportive interaction in the classroom from a multimodal perspective. The support is conceptualised as a joint activity of the participating pupil and teacher. The article analyses in detail how the pupil indicates his need for assistance and the resources used by the teacher in the assistance he gives. In theorising beyond the single case, the article reflects on the rhetorical concept of "support" and on the specifics of interactive assistance in the classroom as a constitutive requirement for the professional activity of teachers.
\end{abstract}

\section{Multimodale Interaktionsanalyse}

Die multimodale Interaktionsanalyse hat ihre Wurzeln zum einen in der konversationsanalytischen Methodologie. Zum anderen ist sie bezogen auf soziologische (Goffman 1983) und anthropologische (Hall 1966; Kendon 1990) Vorstellungen, die bei der Analyse menschlicher Interaktion immer schon neben der Verbalität auch den Gesamtbereich der visuellen Aspekte menschlichen Verhaltens berücksichtigt haben. ${ }^{1}$ Mit solchen Vorstellungen teilt die multimodale Interaktionsanalyse ihre grundlegende theoretische Annahme, dass für die Analyse der schrittweisen Herstellung sozialer Bedeutung alle den Beteiligten zur Verfügung stehenden Ausdrucksressourcen gleichberechtigt berücksichtigt werden müssen. Zu diesen Ausdrucksressourcen gehören Verbalität, Mimik, Gestikulation, Blickverhalten, Körperposituren und Positionen im Raum, die Manipulation von Objekten und unterschiedliche Präsenzmodi. Die multimodale Interaktionsanalyse arbeitet daher ausschließlich mit audiovisuellen Interaktionsdokumenten. Sie trägt damit der faktischen Komplexität von Interaktion Rechnung und fokussiert die eingesetzten Ressourcen einerseits im Hinblick auf ihre fallspezifischen und situativen und andererseits hinsichtlich ihrer allgemeinen Bedingungen und Relevanzen. Mit der Substitution verbaler Interaktionsdokumente durch Videoaufzeichnungen sind hinsichtlich der Gegenstandskonstitution, der theoretischen und methodischen Anforderungen weitreichende Veränderungen verbunden (Schmitt 2006, 2007b, 2007c).

1 Der Ansatz der multimodalen Interaktionsanalyse ist inzwischen im deutschsprachigen Raum mit unterschiedlicher Schwerpunktsetzung gut dokumentiert. Eine gute Orientierung bieten die Bände „Koordination“ (Schmitt 2007c), „Situationseröffnungen“ (Mondada/Schmitt 2010) „Raum als interaktive Ressource“ (Hausendorf/Mondada/Schmitt (Hg.) 2012) sowie die monografische Darstellung „Körperlich-räumliche Aspekte der Interaktion“ (Schmitt im Druck). 
So tritt neben die bei der Analyse verbaler Interaktion im Mittelpunkt stehende Sequenzialität bei Berücksichtigung der visuellen Verhaltensaspekte nun die strukturelle Gleichzeitigkeit interaktionsrelevanter Aktivitäten auf sehr unterschiedlichen Modalitätsebenen. Der „Blick“ auf die faktische Komplexität von Interaktion macht schlagartig die Notwendigkeit deutlich, die verbal definierten Konzepte der Konversationsanalyse auf ihre multimodale Eignung hin zu befragen und neue, gegenstandsadäquate Konzepte zu entwickeln (zum turn-taking siehe beispielsweise Schmitt 2004 und Mondada 2006). Wie verhält es sich beispielsweise mit den Basiskategorien „Sprecher“ und „Hörer“ im multimodalen Forschungszusammenhang (Schmitt 2012b)? Zudem gelangen konstitutive Anforderungen in den Blick, die zuvor bei der Rekonstruktion verbaler Interaktion keine Rolle gespielt haben. Hierzu zählen beispielsweise die Notwendigkeit, sich mit anderen Beteiligten kontinuierlich zu koordinieren (Schmitt 2007a) und die Voraussetzungen für die Durchführung fokussierter Interaktion selbst interaktiv herzustellen. Dies rückt dann allgemeine Verfahren in den analytischen Fokus, mit denen Interaktionsbeteiligte Raum als interaktive Ressource zur Bearbeitung konstitutiver Anforderungen der Interaktionskonstitution herstellen (Hausendorf/Mondada/Schmitt 2012).

Der Bereich der multimodalen Interaktionsanalyse beginnt sich auszudifferenzieren, wobei sich aktuell zwei Schwerpunkte videobasierter Interaktionsanalyse ausmachen lassen. Im Bereich der multimodalen Interaktionsanalyse gibt es einerseits Arbeiten, bei denen es um die multimodale Erweiterung und Präzisierung klassischer konversationsanalytischer Fragestellungen und Konzepte geht: Was passiert beispielsweise bei der turnÜbernahme hinsichtlich Blickorganisation und Gestikulation (Mondada 2004, 2007a, 2007b). Welche Rolle spielen gestikulatorische Aktivitäten bei der Selbst- oder Fremdkorrektur?

Andererseits gibt es Untersuchungen, bei denen der Fokus auf Verbalität keine Primärorientierung bei der Analyse mehr darstellt. Hier geht es vielmehr um allgemeine Fragen der Interaktionskonstitution als komplexes, multimodales Unternehmen. Fragen, die bei einem solchen Erkenntnisinteresse auftauchen, sind beispielsweise die nach der ,Ressourcenqualität von Raum“ (Hausendorf/Mondada/Schmitt 2012; Schmitt 2012b) und die nach „Bewegung als situierter Praxis“ (Schmitt/Deppermann 2010; Hausendorf/Schmitt 2010; Schmitt 2012a).

\section{Unterstützung als pädagogische Anforderung}

Die Unterstützung von Schülern/einzelnen Schülern ist eine der zentralen Anforderungen, denen Lehrer/innen kontinuierlich im Unterricht begegnen, und Teil ihres pädagogischen Auftrags. Relevante pädagogische Begriffe, unter denen in der Didaktik diese Anforderungen diskutiert werden, sind beispielsweise „Lob“, „Ermutigung“, „Rückmeldung“, „Bestätigung“ und Formen der „Fehlerkorrektur“. Diese Begriffe sind zentrale Qualitätskriterien für „guten Unterricht“ (Meyer 2004; Helmke/Schrader 2009). Dabei wird der individuelle Zuschnitt unterstützender Aktivitäten der Lehrer/innen in der Pädagogik unter den Begriffen „Differenzierung und Individuierung“ des Unterrichts behandelt (Bönsch 1995; Kuty 2009).

Wie diese Anforderung jedoch im Unterricht in spezifischen Situationen interaktiv „defacto-didaktisch" (Schmitt 2011a, 2011b) bearbeitet wird und welche unterschiedlichen Verfahren die Lehrer/innen dabei einsetzen, ist im Detail nicht Gegenstand didaktischer 
Forschung. ${ }^{2}$ Dies liegt einerseits am Erkenntnisinteresse der Didaktik, dem keine konstitutionsanalytische Methodologie zugrunde liegt. Andererseits hängt das auch damit zusammen, dass die Art der Unterstützung eines Schülers von sehr unterschiedlichen Aspekten und Bedingungen abhängt. Die konkrete Realisierung der Hilfestellung reagiert beispielsweise auf den Unterrichtstyp (Frontal- versus Gruppenunterricht), die Unterrichtsphase (Stillarbeit, Gruppenarbeit oder fokussierte gemeinsame Arbeit in der Klassenöffentlichkeit), auf den Status und die Kompetenz des Schülers/der Schülerin (guter, durchschnittlicher oder eher schlechter Schüler) und auf die Beziehung, die der Lehrer/die Lehrerin zu dem unterstützungsbedürftigen Schüler hat.

Die Herstellung der Voraussetzung der Unterstützung sowie deren Ausmaß und Vollzugsqualität sind jedoch keinesfalls eine einseitige Angelegenheit des Lehrers. Vielmehr fordern Schüler aktiv Unterstützung ein und verdeutlichen (siehe unten) Ausmaß und Spezifik ihres Unterstützungsbedarfs. Bei der nachfolgenden Analyse wird das fragliche Geschehen daher nicht als Unterstützen im Sinne eines einseitigen Handlungszusammenhangs, sondern als „Unterstützungs-Interaktion“ im Unterricht konzeptualisiert. Damit wird der interaktiven Qualität des Ereigniszusammenhangs Rechnung getragen und die Konstitutionsbeiträge der beiden zentral Beteiligten fokussiert.

Im nachfolgend analysierten Ausschnitt haben wir es mit einem Fall von Unterstützungsinteraktion zu tun, der in mehrfacher Hinsicht interessant ist: Die Unterstützung ist kein lokales, einmaliges Ereignis, sondern erstreckt sich über einen längeren Zeitraum, sie entwickelt sich in unterschiedlichen Phasen mit jeweils eigener interaktions- und situationsstruktureller Spezifik und sie ist darüber hinaus ausgesprochen manifest. Der Lehrer reagiert dabei auf unterschiedliche Aspekte des Schülerverhaltens, und die Unterstützung wird in deutlicher Weise von dem Schüler selbst ,eingefordert" und hinsichtlich ihrer konkreten Realisierung aktiv mitstrukturiert. Im Rahmen einer solchen interaktionistischen Konzeption ist es dann möglich, einzelne unterstützende Verfahren des Lehrers - in ihrer interaktionsstrukturellen Bedingtheit und Sensitivität - als dessen rollenspezifischen und professionellen Konstitutionsbeitrag zur unterstützenden Interaktion herauszuarbeiten. Ich werde im Anschluss zwei Phasen dieser unterstützenden Interaktion zwischen dem Lehrer und einem Schüler im Detail rekonstruieren. Dabei handelt es sich zum einen um eine dyadische Interaktionskonstellation, in der sich die Unterstützungsinteraktion nur zwischen dem Lehrer und dem Schüler entwickelt (Abschnitt 3, Transkript 01-19). Zum anderen untersuche ich eine Unterstützungsinteraktion, die sich in der Öffentlichkeit der Klasse entwickelt und sich daher unter anderen Konstitutionsbedingungen vollzieht (Abschnitt 4, Transkript 27-52).

\section{Unterstützende Interaktion in der Dyade}

Wenden wir uns zunächst dem Kontext zu, in der die Unterstützung als dyadische Interaktion zwischen dem Lehrer und dem einzelnen Schüler entsteht und sich entwickelt.

\subsection{Koordination mit etablierter Interaktion durch Marius}

Der Lehrer (WE) hat sich zwischen Justus (JU) und Marius (MA) platziert und hilft - nach vorne gebeugt und auf das Blatt des Schülers schreibend - Justus bei der Fertigstellung seines Steckbriefes (Bild 1).

2 Zur Bearbeitung dieser Anforderung im Rahmen des Epochenunterrichts in der Waldorfschule siehe Heidtmann/Schmitt (2011). 
Bild 1

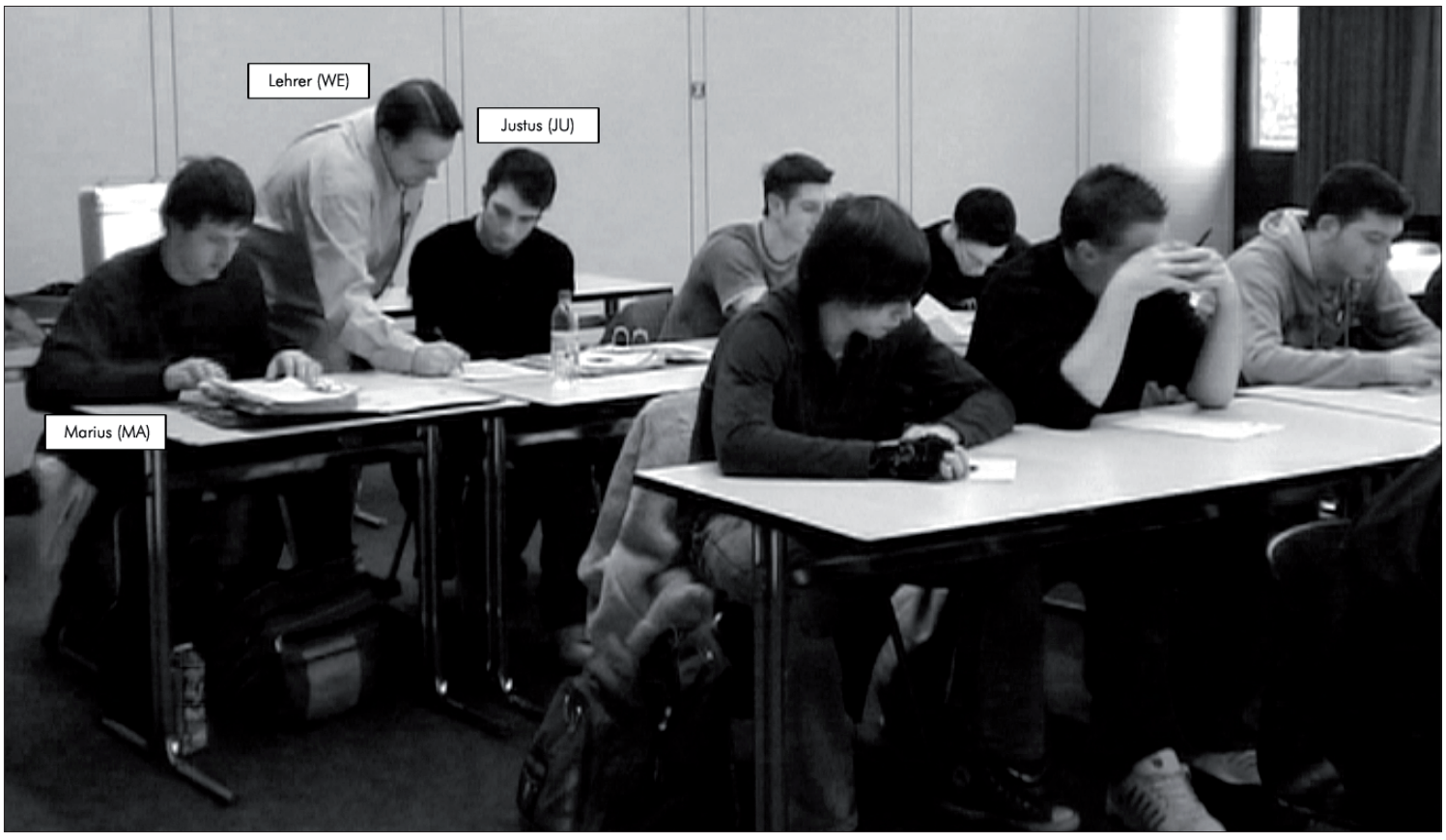

Marius hat seinen Steckbrief etwas früher abgeschlossen und seinen Stift bereits vor sich abgelegt. Er hält sein Blatt mit dem Text leicht angehoben vor sich. Während der Lehrer damit beginnt, den Stift abzulegen, mit dem er das englische Wort ,poisoned“ auf das Blatt des Schülers geschrieben hat, beginnt Marius seinen Kopf nach links zu drehen (Bild 2). Während der Lehrer seine vorgebeugte Positur aufgibt und in den Stand kommt, rückt Marius etwas nach links und damit an den Lehrer heran. Er hat auch sein Blatt mit nach links genommen und es noch etwas angehoben, wodurch es weiter in den Bereich der Wahrnehmungsperipherie des Lehrers rückt. Er schaut zudem auf das Blatt seines Mitschülers bzw. auf den abgelegten Stift (Bild 3).

Bild 2

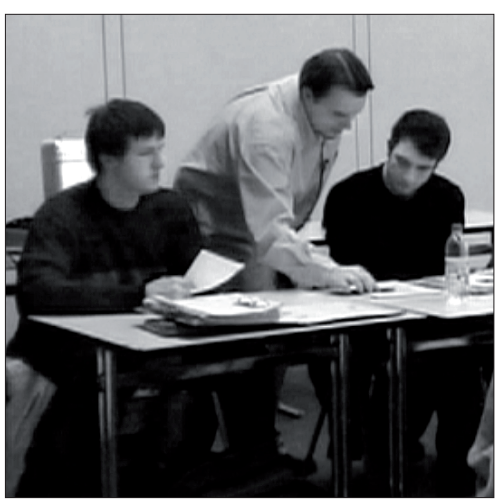

Bild 3

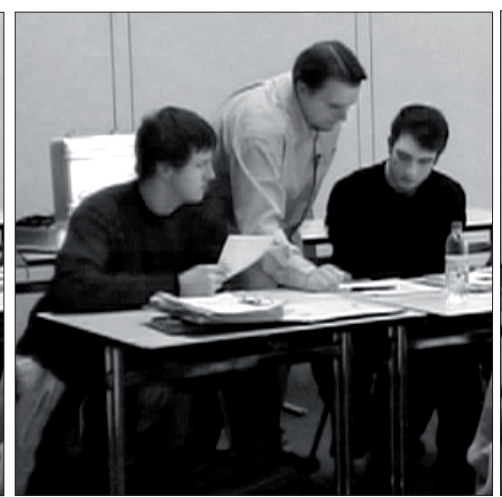

\section{Bild 4}

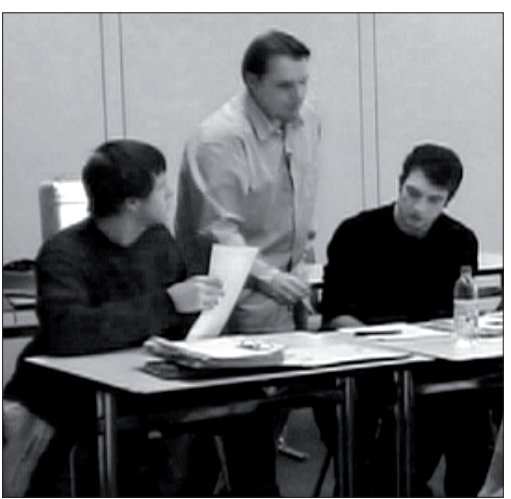

Der Aufwärtsbewegung des Lehrers in den Stand folgt er mit seinem Kopf und Blick. Das Blatt hält er nun noch deutlicher aufgerichtet in seiner rechten Hand (Bild 4). Marius bleibt auch weiterhin auf den Lehrer orientiert und koordiniert sein eigenes körperliches Verhalten mit dessen Bewegungen. Nachdem der Lehrer in den Stand gekommen ist, macht er einen kleinen Schritt rückwärts und dreht sich etwas nach rechts. Sein Blick geht 
zwischen die beiden Schüler, wodurch er wahrnehmen kann, dass sich Marius mit seinem Kopf zu ihm gedreht hat und das Blatt mit dem Steckbrief als „Angebot“ offeriert (Bild 5).

Bild 5

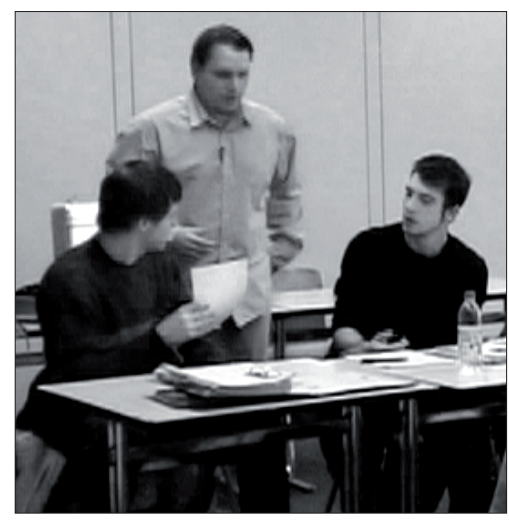

Bild 6

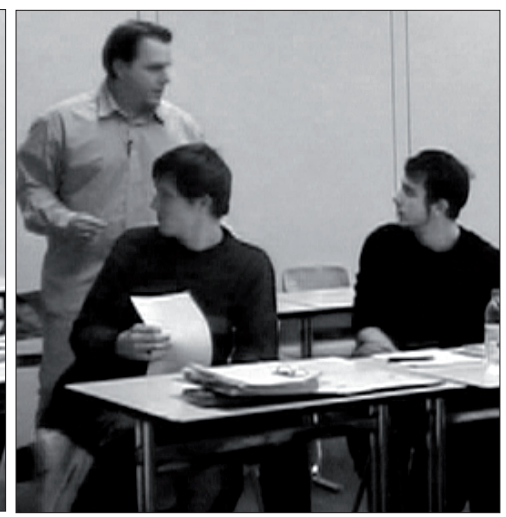

Bild 7

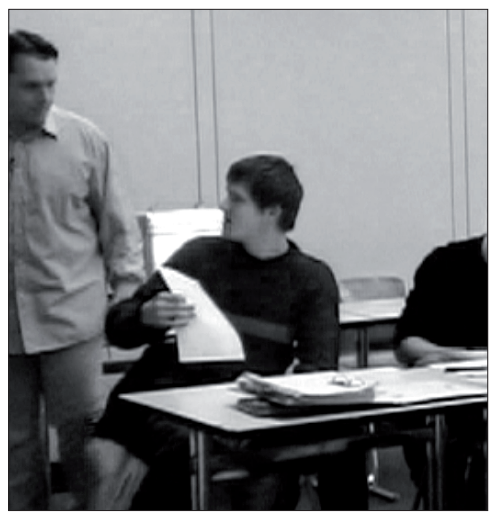

Der Lehrer wendet sich mit seiner letzten Äußerung der ist auch ziemlich gut der film an Justus, der darauf mit Kopfdrehung und Blickorientierung auf den Lehrer reagiert. In die beginnende Abschlussäußerung platziert Marius in Überlappung eine namentliche Adressierung des Lehrers herr weber. Es ist nicht zu entscheiden, ob der Lehrer diese Äußerung gehört hat. Er macht danach einen Schritt nach rechts und damit hinter Marius. Dieser Schritt nach rechts projiziert eine Bewegungsrichtung des Lehrers von der letzten Reihe weg nach vorne. Marius reagiert auf diese Projektion, indem er den Lehrer nochmals namentlich adressiert herr weber und dann nach einer kurzen Pause ein Anliegen formuliert (-) könnten sie das hier mal durchsehen ob da noch fehler drin sind.

Gleichzeitig dreht er seinen Kopf und seinen Oberkörper weit nach rechts. In diese Bewegung nimmt er auch das immer noch aufrecht stehende Blatt mit (Bild 6). Der Lehrer macht einen weiteren Schritt nach rechts und steht nun etwas versetzt hinter dem Schüler, der zu ihm aufblickt und seinen rechten Arm und seine rechte Hand, in der er seinen Steckbrief hält, etwas in die Höhe gehoben hat. Der Lehrer reagiert mit seinem Blick auf die verkörperte Aufforderung des Schülers, auch seinen Steckbrief zu lesen. Es ist nicht eindeutig zu entscheiden, ob der Lehrer Blickkontakt mit Marius hat oder ob er auf dessen Steckbrief schaut (Bild 7). Klar ist jedoch, der Lehrer hat sich inzwischen erkennbar auf Marius orientiert.

Die fein abgestimmte Koordinierung Marius' mit der Abschlussorganisation der Interaktion zwischen Lehrer und Justus und die antizipatorische Orientierung auf den projizierten Laufweg des Lehrers machen deutlich: Der Schüler möchte den Lehrer auf seinem Weg nach vorne auf keinen Fall verpassen, sondern sicherstellen, dass der Lehrer auch einen Blick auf seinen bereits fertigen Steckbrief wirft. Dass der Schüler es nicht bei der Verbalisierung seines Anliegens belässt, sondern durch eine manifeste Verkörperung seine Relevanzen markiert, zeigt die Wichtigkeit, die er dem überprüfenden Blick des Lehrers auf seinen Steckbrief beimisst.

Um den Lehrer auf seinen Text zu orientieren, nutzt Marius ein Verfahren, für dessen erfolgreiche Realisierung die bereits etablierte Unterstützungsinteraktion zwischen dem Lehrer und Justus die zentrale Voraussetzung ist. Nach erfolgter Abschlussorganisation kann Marius gewissermaßen das bereits etablierte Interaktionsmuster „Unterstützung“ für 
sich selbst nutzen und muss dieses nicht erst selbstständig etablieren. Er nutzt die räumliche Nähe des Lehrers und die etablierte Aktivität, obwohl nicht er, sondern Justus den Lehrer mit einer Handmeldung in die letzte Reihe gebeten hat und dockt gewissermaßen an die etablierte Interaktionsstruktur an. Während Justus die Unterstützung des Lehrers bei der Ausarbeitung seines Steckbriefes benötigt, geht es Marius lediglich um die Überprüfung, ob alles richtig ist. Angesichts dieser Tatsache sind der verbale sowie der körperliche Aufwand und die damit verbundene Relevanzhochstufung bemerkenswert. Man kann sich fragen, ob Marius diese Gelegenheit jenseits der Fehlersuche nicht auch dazu nutzt, sich als kompetenter Schüler zu präsentieren und Lob sowie soziale Zuwendung vom Lehrer in einer dyadischen Situation zu erhalten.

Beim „Andocken“ handelt es sich um ein Verfahren, das man systematisch gerade in Unterrichtssituationen beobachten kann. Heidtmann/Schmitt (2011, S. 197ff.) beschreiben im Rahmen ihrer Analyse zum ,interaktiven Diktieren“ in einer Waldorf-Epoche beispielsweise einen Fall, bei dem ein Schüler an das zuvor etablierte und bereits zweimal realisierte Muster des Nachfragens andockt. Mit „Andocken“ wird eine spezifische Form schülerseitigen Anschlusshandelns konzeptualisiert, für das folgende Aspekte konstitutiv sind: die Nutzung bereits bestehender und von anderen Beteiligten etablierten thematischpragmatischen Relevanzen und Aktivitätstypen sowie teilweise auch interaktionsstrukturellen Konstellationen, um eigene Relevanzen zu bearbeiten. Andocken kann sehr unterschiedliche Formen annehmen und dabei in kontextsensitiver Weise sehr unterschiedliche Ressourcen einsetzen. Es kann ausschließlich verbal abstinent realisiert werden. Voraussetzung hierfür ist jedoch in der Regel die räumliche Nähe des Adressaten. Es kann - vor allem, wenn es auf Distanz hin realisiert wird, wie das im oben zitierten Beispiel der Fall ist - auch primär auf Verbalität zurückgreifen. Für beide Realisierungsformen ist vorgängiges detailliertes Monitoring des andockenden Schülers zur Identifikation des richtigen Zeitpunktes und der Wahl der einzusetzenden Ressourcen die zentrale Voraussetzung. ${ }^{3}$

Der Lehrer macht noch einen Schritt nach vorne und kommt dann fast auf einer Höhe mit dem sitzenden Schüler zum Stand. Er hat sich inzwischen dem Schüler so stark genähert, dass sich seine linke Hand mit der rechten Hand des Schülers, in der dieser das Blatt mit dem Steckbrief hält, fast berührt. Der Blick des Lehrers geht nun auf das Blatt, das Marius nochmals etwas angehoben hat.

Bild 8

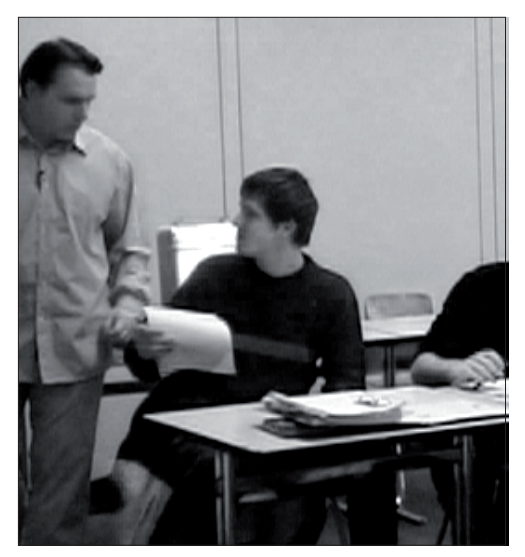

Bild 9

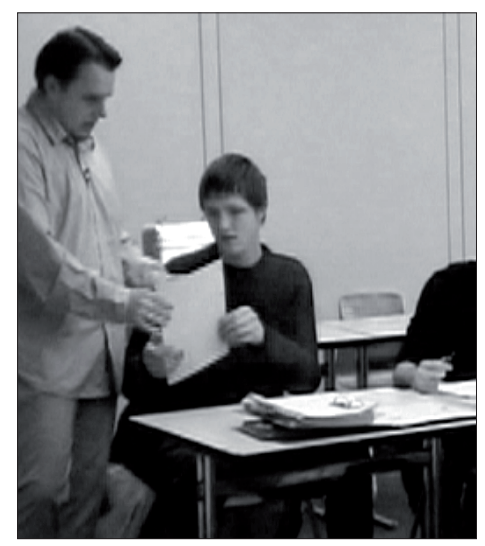

Bild 10

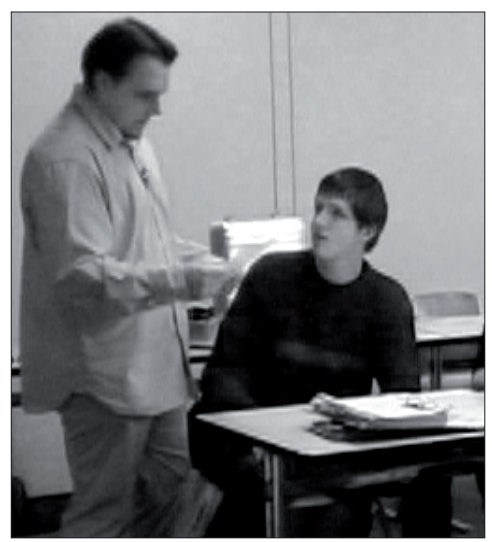

Es ist davon auszugehen, dass das Konzept nicht nur im Unterricht von Schülern praktiziert wird, sondern als generelles, letztlich situationsunabhängiges Verfahren zum Einsatz kommt. 
Marius selbst behält seine leicht nach oben gerichtete Kopfhaltung zunächst weiter bei (Bild 8). Als Zeichen der vollständigen Orientierung auf den Schüler dreht sich der Lehrer in der Hüfte etwas nach links ein und ergreift mit beiden Händen das vom Schüler präsentierte Blatt. Beide blicken nun gemeinsam auf den Steckbrief und realisieren damit eine punktgenaue Fokussierung. Zudem halten beide - als weitere Form einer kollaborativen Aktivität - das Blatt für einen kurzen Moment mit beiden Händen senkrecht (Bild 9).

Nachdem sich der Lehrer stabil bei dem Schüler positioniert und durch das „Einrasten“ für die Durchführung fokussierter Interaktion ein stabiles Fundament (Müller/Bohle 2007) geschaffen hat, lässt Marius das Blatt los. ${ }^{4}$ Er hat beide Hände unterhalb der Tischplatte abgelegt und wendet auch seinen Blick von dem Blatt. Er verfolgt nun, in das Gesicht des Lehrers blickend, wie dieser beginnt, seinen Text zu lesen (Bild 10). Indem der Schüler sein bisheriges unterstützungseinforderndes Verhalten einstellt, schafft er für die Lektüre seines Textes durch den Lehrer optimale Bedingungen.

3.2 Lektüre des Steckbriefes und Positivevaluation

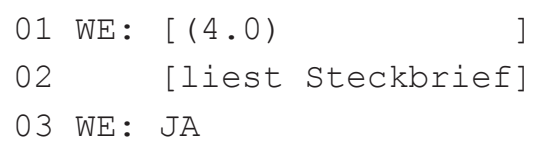

Der Lehrer liest für vier Sekunden den Text des Schülers. Marius hält während dieser Phase seinen Blick unverändert auf das Gesicht des Lehrers geheftet, wodurch es ihm möglich ist, die Mimik und die sich eventuell darin ausdrückende Einschätzung seines Textes wahrzunehmen.

Der Lehrer beendet seine Lektüre mit einem positiv evaluierenden JA (Z. 03). Sein positives Lob ist eine für Lehreraktivitäten typische ,third position activity“ (Nassaji/Wells 2000; Lee 2007), die zwei vorgängige Aktivitäten voraussetzt: eine Aufgabenstellung des Lehrers und die Bearbeitung des Schülers (hier der Steckbrief). ${ }^{5}$

\subsection{Implizite Korrektur}

Im direkten Anschluss leitet er dann mit allerdings eine relativierende Äußerung ein.

04 WE: allerdings des einzige was (---)

Er bringt diese Äußerung jedoch nicht zu Ende, sondern unterbricht deren Produktion zunächst mit einer Pause (---). ${ }^{6}$ Fragt man danach, welches Element an der mit der Pause „ausgesparten“ Position strukturell erwartbar ist, so verzichtet der Lehrer auf die Formulierung eines explizit negativen Hinweises, der die Fehlerhaftigkeit eines Textelements explizit benennt: das einzige was (,nicht stimmt“ oder „,falsch ist“" wäre hier einzusetzen). Gleichzeitig weist das einzige darauf hin, dass es sich nur um ein einziges Element handelt, sodass sich die Relativierung nicht auf den gesamten Text, sondern nur auf ein Detail bezieht. Mit Beginn dieser Äußerung gibt er seine stehende Position auf, beugt sich nach vorne und legt das Blatt mit dem Steckbrief auf dem Tisch des Schülers ab. Marius löst

4 Zur Herstellung der körperlich-räumlichen Voraussetzungen für die Eröffnung fokussierter Interaktion siehe Mondada/Schmitt (2010).

$5 \mathrm{Zu}$ diesem Mechanismus der Unterrichtskonstitution siehe beispielsweise McHoul (1978), Mehan (1979), Sinclair/Coulthard (1975), van Lier (1988), Lemke (1990), Markee (2004) sowie Lee (2007).

6 „Pause“ ist im Sinne von Schmitt (2004) immer als „Sprechpause“ zu verstehen. 
Ebenfalls mit Beginn dieser Bewegung des Lehrers seinen Blick von dessen Gesicht und folgt dem Blatt auf seinem Weg auf die Tischplatte.

Bild 11

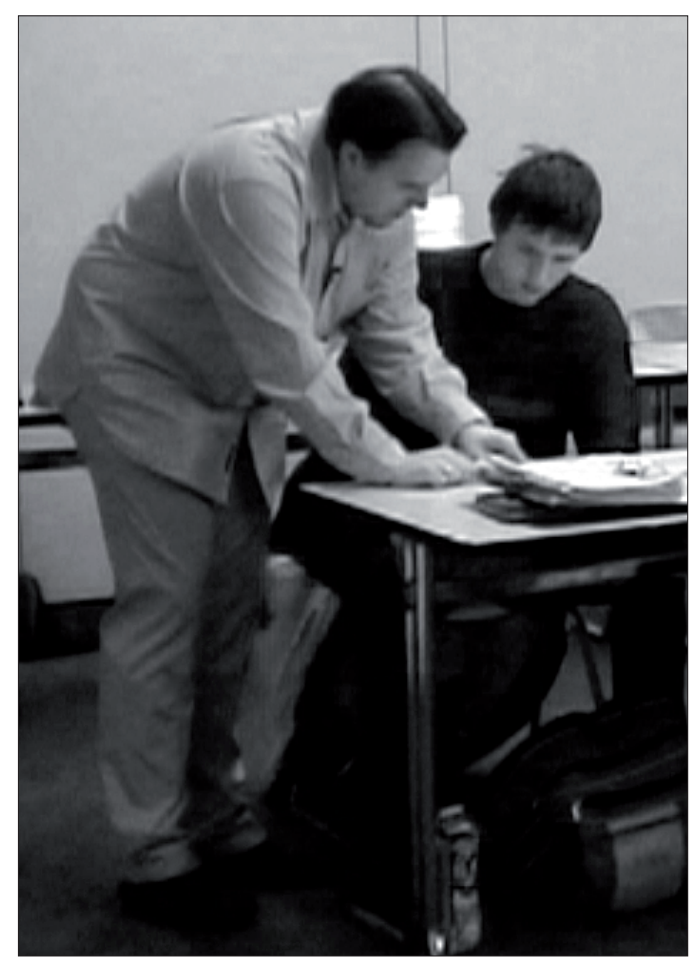

05 WE: many (1.0) much äh ${ }^{7}$

Der Lehrer beendet die Pause mit many, wobei er die Äußerungsentwicklung auch hier gleich wieder einstellt und zunächst eine erneute Sprechpause macht (1.0). Er nimmt die Äußerungsentwicklung dann mit much äh wieder auf. Als der Lehrer in diesem Zusammenhang mit dem Zeigefinger seiner linken Hand den Text des Schülers berührt, der bereits auf dem Tisch liegt, kann Marius diese fokussierende Gestikulation des Lehrers verfolgen (Bild 11). ${ }^{8}$ Beide sind nun in ihrer Körper- und Blickausrichtung parallelisiert und fokussieren gemeinsam den Text von Marius und die zeigenden Aktivitäten des Lehrers.

Damit ist nun klar: Der Lehrer korrigiert hier einen grammatischen Fehler, den Marius bei seinem Steckbrief gemacht hat. Er hat - so können wir schließen - fälschlicherweise much goals geschrieben statt many goals. Interessant ist bei dem verbalen Verhalten des Lehrers eine durchgehende Vermeidung der expliziten Formulierung der Fehlerhaftigkeit des Steckbriefes und der Benennung des Schülers als Urheber dieser Fehlerhaftigkeit. Dies wird nicht nur im Auslassen eines negativen Elements deutlich, sondern auch im deskriptiven Modus des Hinweises des is also many goals (Z. 06). Er sagt nicht etwa „hier musst

7 Die Abbildungen sind den fett markierten Transkriptstellen zugeordnet.

8 Wir haben es hier mit einer gestikulatorischen Aktivität zu tun, bei der der Lehrer nicht nur - wie bei den „environmentally coupled gestures“ (Goodwin 2007) - einen gemeinsamen Wahrnehmungsfokus markiert, sondern in diesem geteilten Fokusbereich relativ zu den etablierten thematisch-pragmatischen Relevanzen für den Schüler verdeutlichend aktiv wird. 
du many schreiben“ oder „much ist falsch, da muss es many heißen“. Als der Lehrer mit goals das zentrale Textelement nennt, das grammatisch korrekt many statt much fordert, löst er seinen Blick vom Blatt und blickt Marius direkt ins Gesicht.

\section{Bild 12}

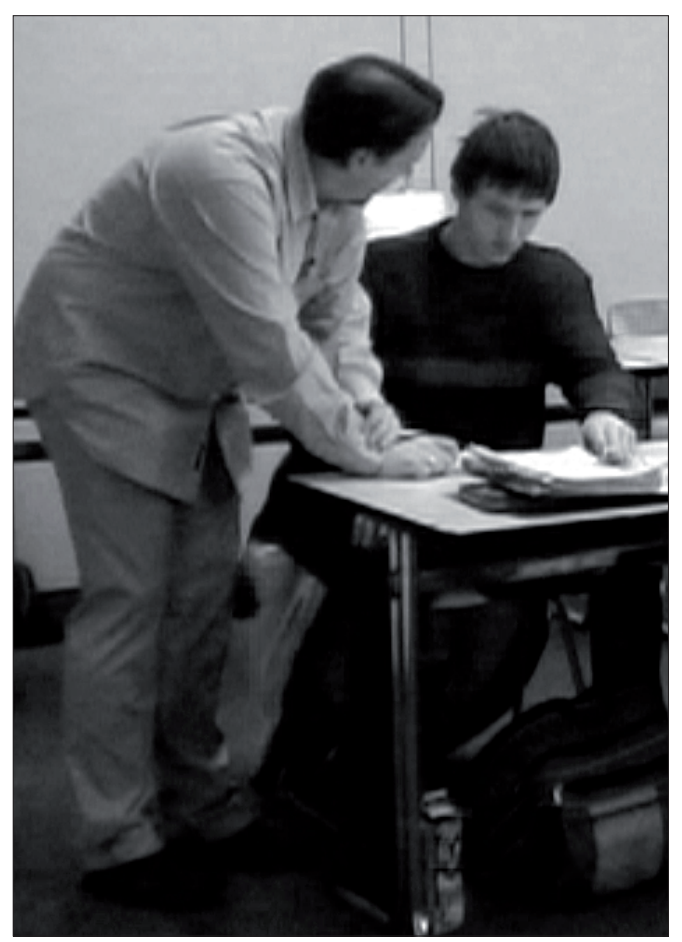

$06 \mathrm{WE}$ : des is also many goals ja

Er hat in der Zwischenzeit seine zuvor zeigende linke Hand auf seiner rechten abgelegt und gibt damit den Blick auf den Text für Marius frei (Bild 12). Hier steht der Blickwechsel in erster Linie im Dienste der intersubjektiven Absicherung des gemeinsamen thematischen Fokus und ist daher Teil des lehrerseitigen Monitorings. Der Blick zielt auf die Überprüfung des schülerseitigen Verstehens und ,sucht“ in diesem Sinne nach einer Verstehensdokumentation (Deppermann/Schmitt 2008; Schmitt 2010).

\section{$3.4 \quad$ Regelformulierung}

Der Lehrer beginnt dann eine Äußerung, die mit dem Anschluss wegen dem die direkte Fortführung der vorherigen Äußerung und eine Begründung oder Erläuterung als Folgeelement erwartbar macht. 


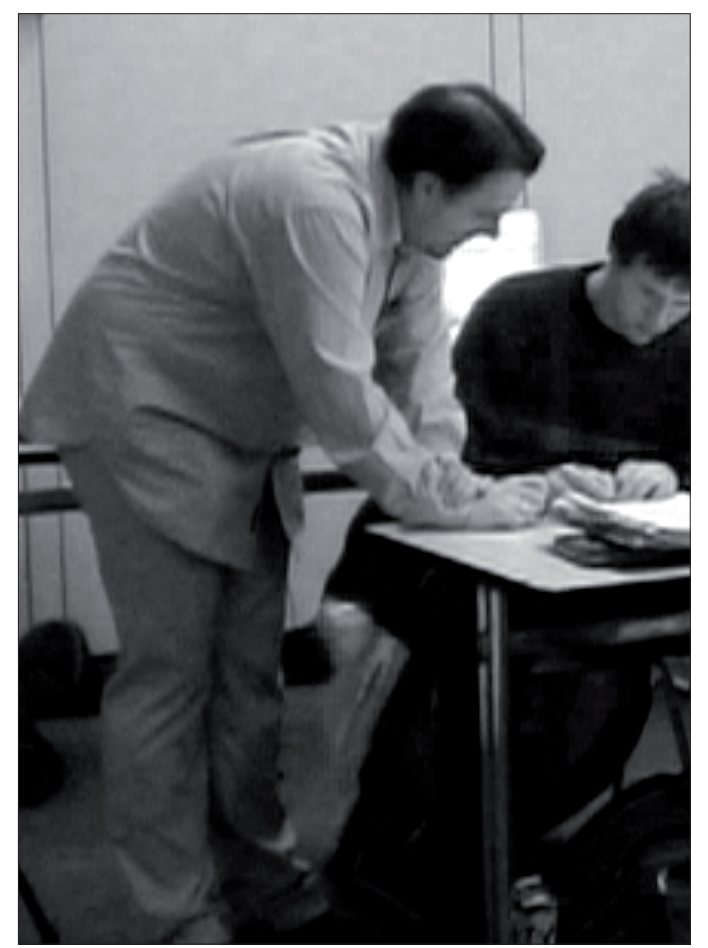

Bild 13

Dieser Projektion folgt der Lehrer jedoch nicht, sondern beginnt mit wenn man sie eine neue Äußerung, die deutlich als Neustart konzipiert ist. Er behält die Eröffnungsstruktur wenn man der Äußerung bei der nachfolgenden Fortsetzung wenn man die sachen zählen kann bei. In dieser neuen Äußerung wird jedoch die Substitution eines Elementes deutlich: sie wird durch die sachen ersetzt. Das ist insofern interessant, als damit auch ein Wechsel des konkreten Bezuges auf den Steckbrief und die „vielen Tore“ (wenn man sie zählen kann) zu einem abstrakten, falltranszendierenden Bezug auf „Sachen“ allgemein einhergeht. Der Lehrer hat seine Handstellung beibehalten und verfolgt konzentriert, wie Marius beginnt, seinen Text nach den vorangegangenen Ausführungen zu korrigieren (Bild 13). Die eröffnete wenn-dann-Struktur wird mit der Fazit-Formulierung dann heißt es many abgeschlossen. Man kann in diesem schrittweisen Umbau der Regelformulierung, denn genau darum handelt es sich hier, einen Aspekt von ,recipient design“ (Sacks 1992; Sacks/Schegloff/Jefferson 1974; Malone 1997, S. 100-119; Schmitt/Deppermann 2009) erkennen. Der Lehrer arbeitet sich schrittweise auf eine - aus seiner Perspektive für Marius passende bzw. auf ihn speziell zugeschnittene Realisierung der Regel für den Gebrauch von much und many hin. 


\subsection{Fallbezogene Exemplifizierung}

Nach der allgemeinen Regelformulierung bezieht sich der Lehrer dann nochmals auf den konkreten Steckbrief zurück.

\section{Bild 14}

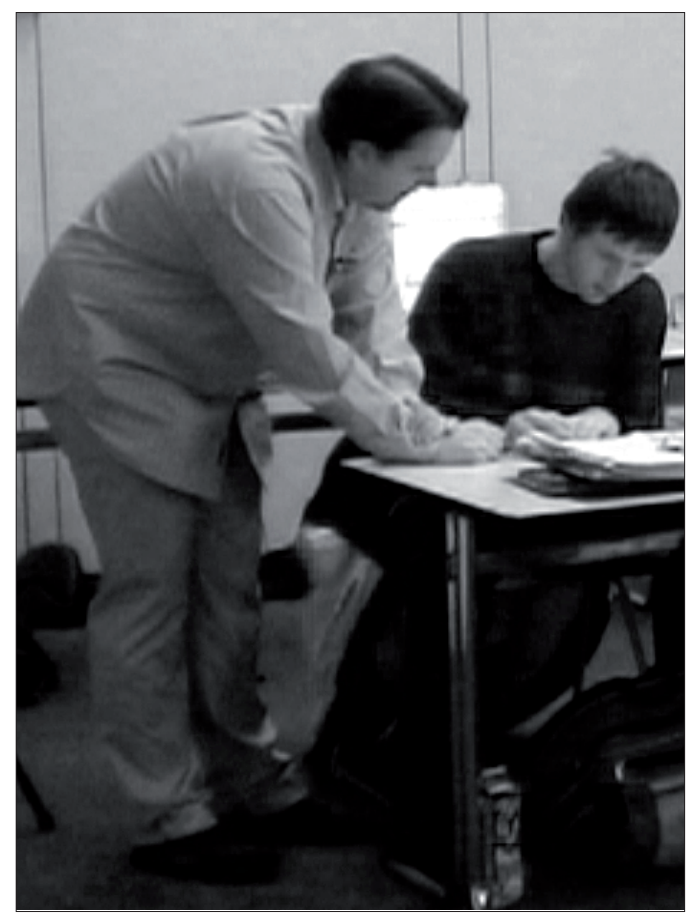

11 WE: und tore kann man ja zählen

12 ne?

$13 \mathrm{MA}: \mathrm{ja}$

$14 \mathrm{WE}: \mathrm{ja}$.

Der fallspezifische Hinweis und tore kann man ja zählen lässt sich problemlos an die zurückliegende Äußerung des Lehrers des is also many goals (Z. 06) rückbeziehen. Beide Äußerungen hätten ausgereicht, um den falschen Gebrauch von much und den richtigen Gebrauch von many zu erläutern. Durch den Bezug auf das Konzept „recipient design“ erhalten wir hier wichtige Informationen hinsichtlich der Leistungseinschätzung und der Verstehensvoraussetzungen des Schülers aus Sicht des Lehrers. Beginnend mit seiner Äußerung dreht er seinen Kopf nach links und blickt Marius, der weiterhin mit der Korrektur seines Textes beschäftigt ist, ins Gesicht (Bild 14). Der Lehrer initiiert dann mit der tag question ne? eine explizite Ratifikation des Schülers und ,zwingt“ diesen dadurch zu der Verstehensdokumentation ja, die der Lehrer seinerseits mit einem ja (Z. 14) ratifiziert. ${ }^{9}$

9 Das Einfordern und die Überprüfung solcher Verstehensdokumentationen gehört zu den kategoriengebundenen Aktivitäten der Lehrer/innen. Interessant ist dabei, dass sie sich nicht primär oder ausschließlich auf das verbal formulierte Verstehen stützen, sondern sich - sozusagen - durch visuelles Monitoring - im wahrsten Sinn des Wortes - ein eigenes Bild machen. 


\subsection{Rechtschreibhilfe und Ratifikation}

Marius realisiert mit many im Anschluss an die vorherige Initiierung der Verstehensdokumentation eine zweite Verstehensdokumentation, die in sehr unspezifischer Weise signalisiert, dass er verstanden hat, was das Problem bei seinem Steckbrief ist.

\section{Bild 15}

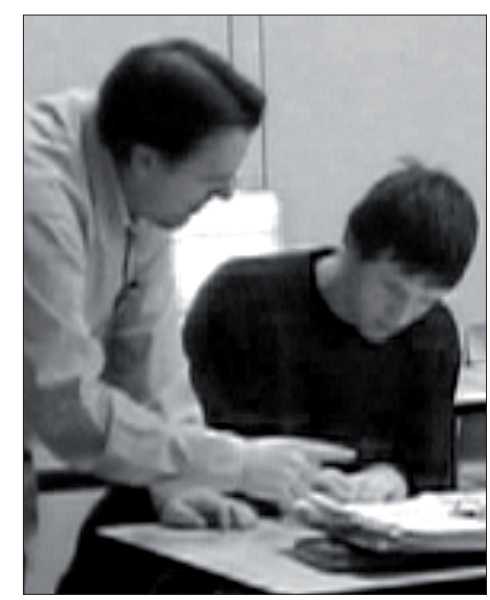

Bild 16

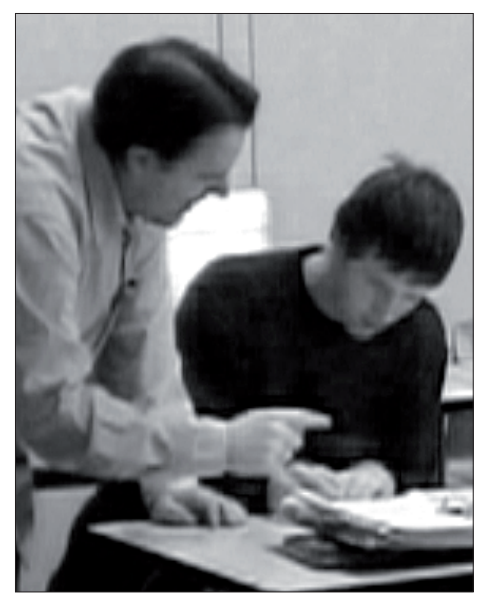

Bild 17

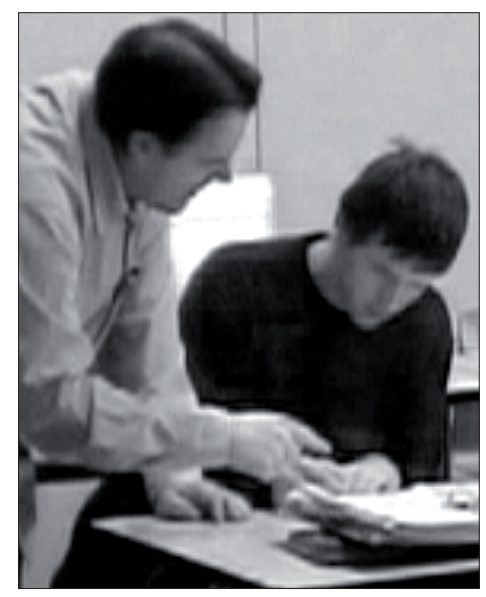

$15 \mathrm{MA}:$ many

16 WE: nä: <<buchstabiert>m m a $\mathbf{n} \mathbf{y}>$

17

(0.5) ja genau

Es ist nicht möglich, aus dem many zu schließen, was genau Marius verstanden hat: dass an der Stelle in seinem Steckbrief much durch many ersetzt werden muss, oder ob er die Regel der Verwendung von much und many verstanden hat. Der Lehrer kontrolliert die konkreten Korrekturaktivitäten des Schülers, der damit beginnt, in seinem Text die Ersetzung vorzunehmen. Das nä: des Lehrers ist ein Hinweis darauf, dass es mit der orthografischen Realisierung der Ersetzung Probleme gibt. Dies veranlasst ihn, die korrekte Schreibweise durch Buchstabierung des Wortes sicher zu stellen. Er begleitet das Buchstabieren mit kleinen Akzentsetzungen seines rechten Zeigefingers (Bild 15-17). Nachdem Marius die Ersetzung erfolgreich und in korrekter Schreibweise realisiert hat, beginnt der Lehrer seine Position bei dem Schüler aufzulösen.

\subsection{Positive Abschlussevaluation}

Der Lehrer beginnt seine Wegorientierung von Marius und damit die Auflösung der fokussierten dyadischen Interaktion mit dem Schüler mit einem expliziten positiven Lob das ist schön. Bei schön tippt der Lehrer zweimal kurz mit seiner linken Hand lose auf die Tischplatte (Bild 18). Er ist bereits fast vollständig zum Stand gekommen und hat seinen rechten Arm bereits deutlich von der Tischplatte entfernt. Auch seine linke Hand, die sich noch auf der Tischplatte befindet, hat der Lehrer bereits partiell angehoben, sodass sich sein Handballen bereits in der Luft befindet. Seinen Blick hat er jedoch weiterhin auf Marius oder dessen Unterlagen gerichtet. 


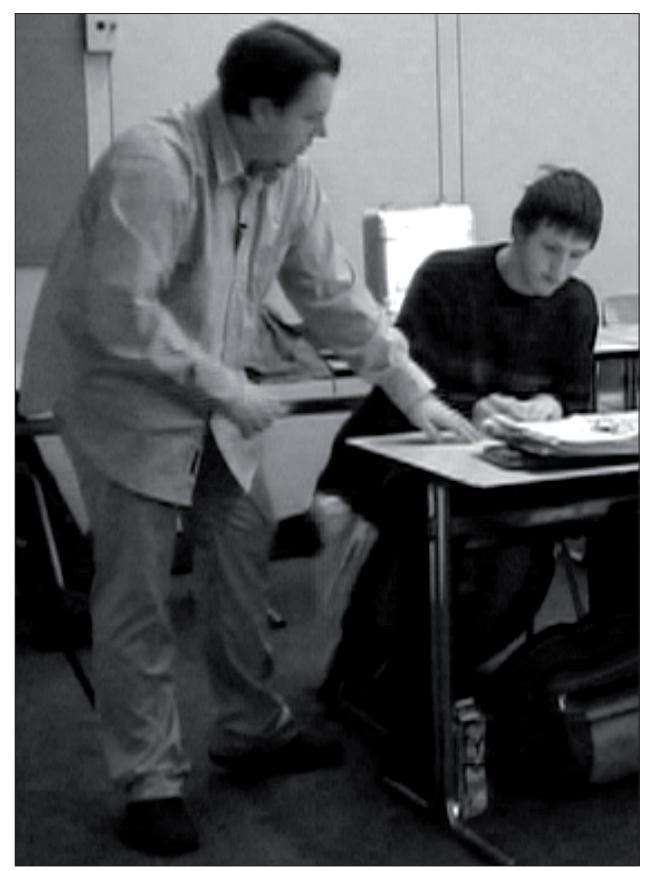

18 WE: das ist schön (.)

\subsection{Vororientierung des Schülers}
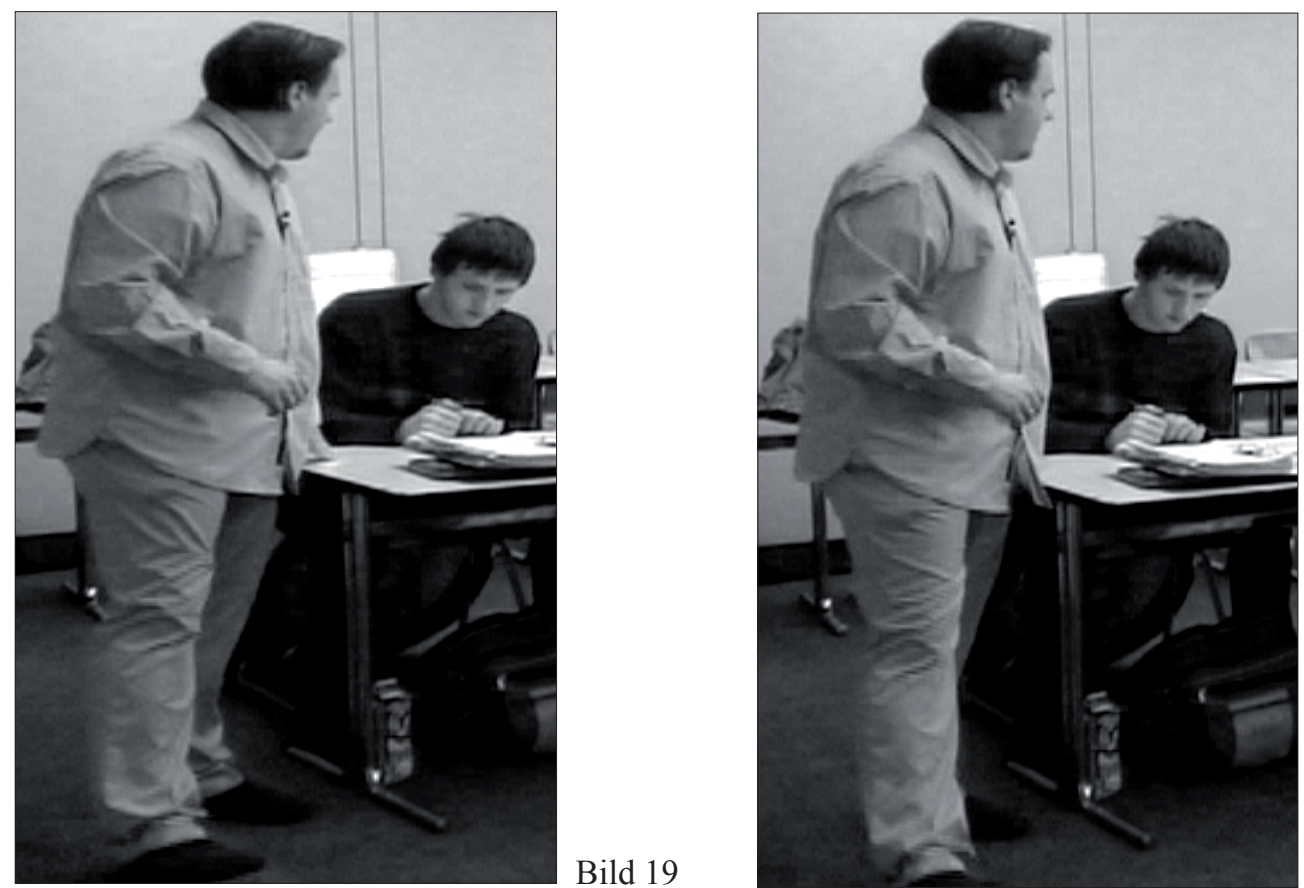

Bild 20

9 WE: kannste vorlesen auch (-) ja? 
Als Beendigung seiner Unterstützung bereitet er den Schüler nach einer ganz kurzen Pause mit dem Hinweis kannste vorlesen auch (Bild 19, 20) darauf vor, dass er ihn zum Vorlesen seines Steckbriefes aufrufen wird. Geht man davon aus, dass alle Schüler ihre Steckbriefe vorlesen können, bzw. es Sinn der Stillarbeitsphase ist, Steckbriefe für die Präsentation zu erstellen, dann ist der Hinweis des Lehrers, dass Marius seinen Steckbrief vorlesen kann, nur als spezielle Vorankündigung zu deuten.

Beendet wird die dyadische Interaktion mit Marius verbal mit der tag question ja?, mit der der Lehrer das Vorankündigungspotenzial seines Hinweises kannste vorlesen auch verstärkt. Beginnend mit ja? löst der Lehrer seinen Handkontakt mit der Tischplatte des Schülers und beginnt nunmehr auch seinen Blick abzuwenden. Er verlässt die Position bei Marius und beginnt seinen Gang in den vorderen Teil des Klassenzimmers. Dabei schaut er in signifikanter Weise - etwas zu lange, um sich nur selbst zu orientieren - auf seine Armbanduhr (Bild 21-23).

Bild 21

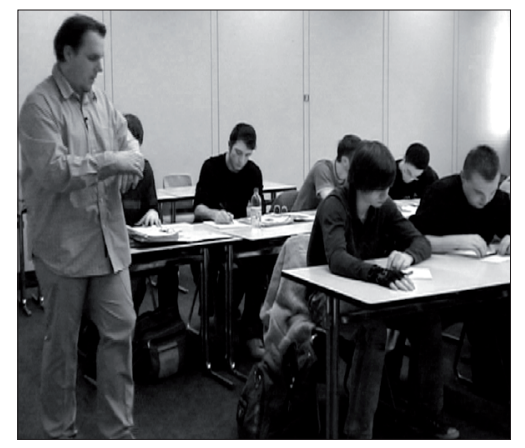

Bild 22

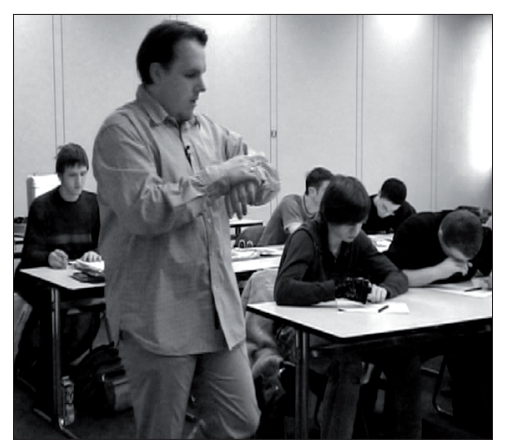

Bild 23

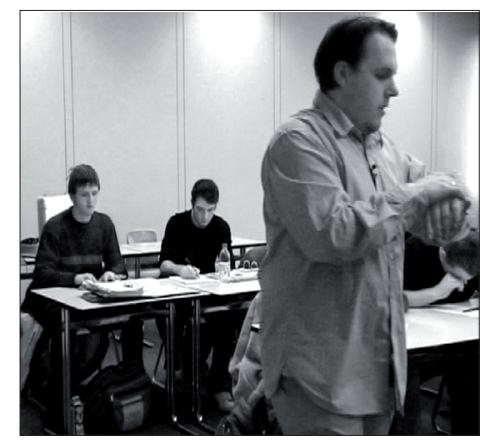

$20 \mathrm{WE}:[(3.2)$

21 [geht nach vorne zur ersten Tischreihe

Er löst seinen Blick von der Armbanduhr erst, als er an der ersten Tischreihe vorbei nach links in Richtung Tafel und Lehrerpult schwenkt. Der Blick auf die Uhr ist so auch für die Wahrnehmung durch die Schüler zeitlich gestreckt. Die Tatsache, dass der Lehrer nicht überprüft, ob sein Blick auf die Uhr tatsächlich auch wahrgenommen wird, ist wiederum ein Hinweis darauf, dass er sich selbst als Fokusperson ${ }^{10}$ und damit als permanent unter Wahrnehmung stehend betrachtet.

\subsection{Analyse-Resümee (1)}

Die Analyse der dyadischen Unterstützungsinteraktion hat in grundsätzlicher Weise das Zusammenspiel von Schüler und Lehrer bei der Etablierung und der Realisierung der Unterstützung gezeigt.

10 Das Konzept „Fokusperson“ wird in Schmitt/Deppermann (2007) und (2010) skizziert und weiter ausgeführt in Schmitt (2010) und Schmitt (im Druck). Es ist ein Beispiel für die Notwendigkeit, für die multimodale Interaktionsanalyse gegenstandsadäquate Konzepte zu entwickeln, die der audio-visuellen Spezifik der zugrundeliegenden Interaktionsdokumente Rechnung tragen. 


\section{Initiierung der Unterstützungsinteraktion durch den Schüler}

Der Schüler nutzt bei der Verdeutlichung seines Unterstützungsbedarfs die bereits etablierte und in seiner unmittelbaren Nähe stattfindende Unterstützungsinteraktion zwischen Justus und dem Lehrer. In einem Verfahren des „Andockens“ zeigt er dem Lehrer Unterstützungsbedarf nicht nur verbal, sondern auch körperlich und durch die präsentative Manipulation des Blattes, auf dem sein Steckbrief steht. Es ist vor allem die frühzeitige Koordination (Schmitt 2007a) mit dem Verhalten des Lehrers, die Konstanz des Monitorings, die Antizipation des Folgegeschehens (wo wird der Lehrer als Nächstes hingehen) und die Dauerhaftigkeit und Systematik der blicklichen und körperlichen Orientierung auf den Lehrer sowie die zweifache namentliche Adressierung des Lehrers, die dem Verhalten von Marius unter den gegebenen Bedingungen einen deutlich auffordernden Charakter verleihen. Hierdurch etabliert er auf der Basis der unterrichtsspezifischen Rollen- und Aufgabenstruktur für den Lehrer eine verstärkte Reaktionsverpflichtung.

\section{Unterstützung des Lehrers}

Der Lehrer adaptiert seine Reaktion auf die mit der verkörperten Unterstützungsbedürftigkeit verbundene Selbstpositionierung des Schülers als vorsichtig, zurückhaltend und eher unsicher. Er realisiert darauf bezogen seine Unterstützung mit einem klaren ,recipient design", das zum einen die relevanten Aspekte der situativen schülerseitigen Selbstpräsentation verarbeitet. Zum anderen wird das ,recipient design“ auch aus dem situationsübergreifenden Wissen des Lehrers über den Schüler gespeist. Dadurch gewinnt das situative Verhalten die Qualität eines „Dokumentes“ im Sinne einer dokumentarischen Interpretation. ${ }^{11}$ Alle unterstützenden Aktivitäten des Lehrers konvergieren unter dieser Perspektive: die vorgreifende unspezifische Evaluation, die implizite Form der Korrektur des grammatischen Fehlers, die auf den Schüler zugeschnittene Regelformulierung, die Überprüfung der korrekten Schreibweise und das gestikulationsbegleitende Buchstabieren sowie die positive und explizite Abschlussevaluation und die Vororientierung des Schülers auf das Vorlesen seines Steckbriefes.

Insgesamt zeichnet sich das unterstützende Verhalten des Lehrers durch einen hohen interaktiven Aufwand aus. Dieses Ausmaß reflektiert als „recipient design“ den Status des Schülers bzw. zeigt die faktische Statuszuschreibung des Lehrers. Weiterhin wird eine handlungsleitende Orientierung des Lehrers deutlich, die Unterstützung des Schülers über die vollständige Bearbeitung inhaltlicher Fehler und Unklarheiten zu stellen. Dabei steht das körperliche Verhalten des Lehrers im Dienste der deutlichen und kontinuierlichen Symbolisierung seiner Orientierung auf und Koordinierung mit Marius.

\section{Unterstützen in der Öffentlichkeit der Klasse}

Die zweite Phase schließt direkt an den analysierten Ausschnitt an. Sie zeigt eine unterstützende Interaktion zwischen dem Lehrer und Marius unter veränderten interaktionsstrukturellen Bedingungen. Hatten der Lehrer und Marius zuvor eine Interaktionsdyade konstituiert, erfolgt die Unterstützung nunmehr in der Öffentlichkeit der Klasse.

11 Zur dokumentarischen Methode der Interpretation vgl. Mannheim (1952) und Garfinkel (1967). 


\subsection{Ankündigung der Steckbrief-Präsentationen}

Der Lehrer beginnt mit der Ankündigung der Steckbriefpräsentationen, als er nach der ersten Tischreihe nach links in Richtung Lehrerpult einbiegt: $\langle<f>$ okay folks we have ten minutes (1.0) $\ddot{a}: h m$ I would like to: äh hear AS many descriptions As possible; hh and I already saw some of them a:nd they are very different $>$ (Z. 22-26).

Am Ende seiner Ankündigung steht er neben dem Pult in der Position, von der aus er in aller Regel die Interaktion mit der Klassenöffentlichkeit führt und von wo aus er organisatorische Hinweise gibt (Bild 24).

\section{Bild 24}

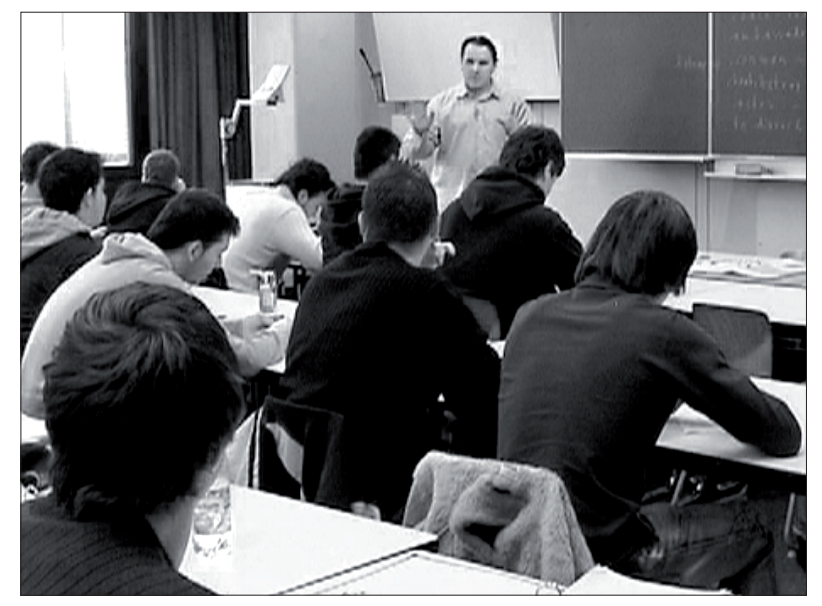

\subsection{Aufruf von Marius}

Der Lehrer dreht sich nach links, blickt in Richtung Marius und fordert diesen auf, als erster Schüler seinen Steckbrief vorzulesen.

Bild 25

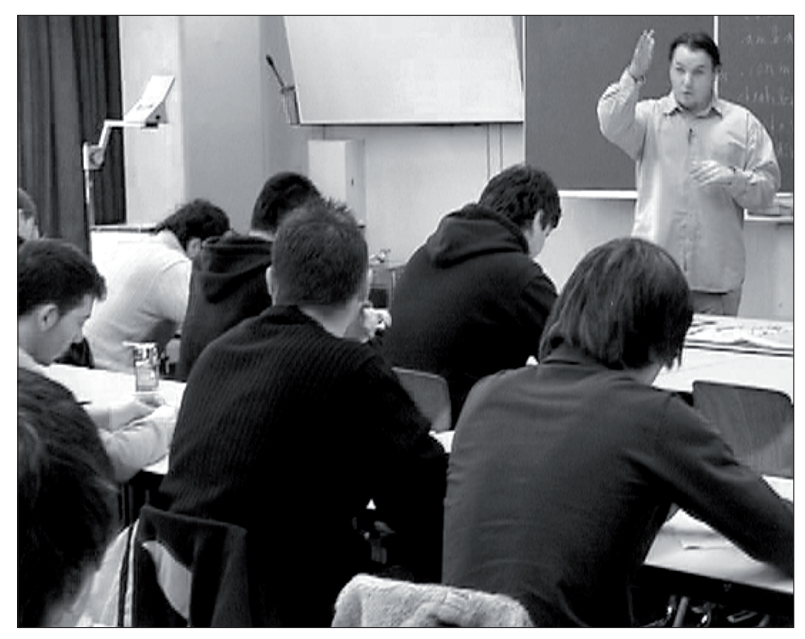

27 WE: MArius (.) would you like to start REAding? 
Neben der Ausrichtung seiner Körpervorderseite auf Marius unterstreicht er seine Orientierung auf den Schüler mit einer manifesten Zeigegeste in Marius' Richtung (Bild 25). Diese wird besonders dadurch manifest, weil sie sich beim Erreichen ihrer maximalen Höhe auf Kopfhöhe des Lehrers befindet. Auch mimisch wird die Orientierung auf Marius durch eine gekräuselte Stirn verdeutlicht. Der Lehrer bringt hier also beim Aufruf des Schülers ein ganzes Bündel modalitätsspezifischer Ressourcen zum Einsatz. In der anschließenden Pause (3.5) setzt der Lehrer seine mit der an Marius gerichteten Aufforderung zum Vorlesen begonnene Bewegung nach links fort, wobei er seinen Blick kontinuierlich auf den Schüler gerichtet hält. Er hat - zum Zeichen des Wartens auf den Präsentationsbeginn - beide Hände lose vor seiner Brustmitte ineinander verschränkt (Bild 26).

Doch nicht nur er ist auf Marius orientiert, sondern auch der Schüler links im Bild hat sich in Erwartung der beginnenden Präsentation zu Marius umgedreht.

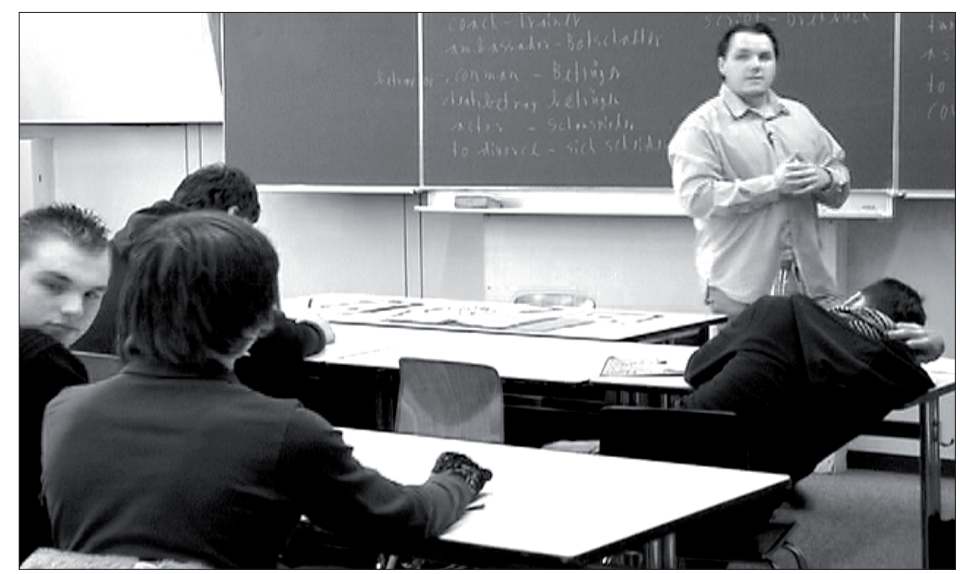

\section{$28 \quad$ (3.5)}

$29 \mathrm{WE}: \quad<\langle\mathrm{h}>\mathrm{mh}$ ? $>$

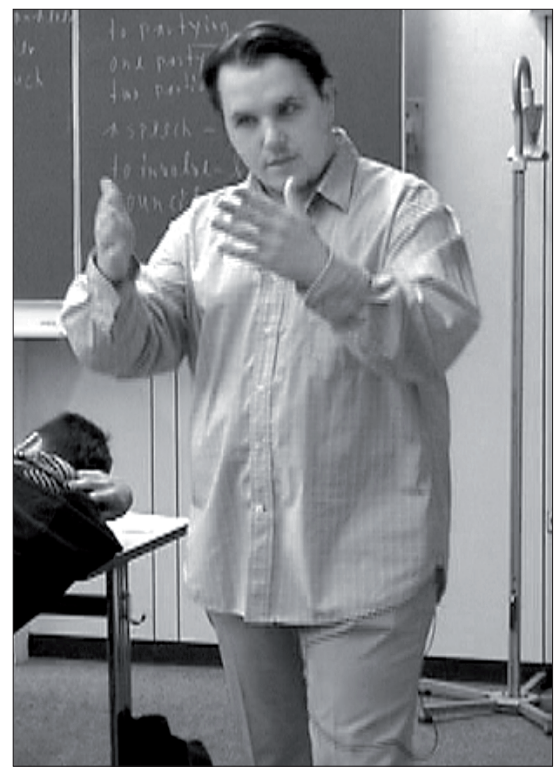

Bild 27

30 MA: <<p>soll ich den $[(\ldots)]$ ?>

$31 \mathrm{WE}: \quad$ [you] just just READ what you wrote; 
Da Marius nicht sogleich mit dem Vorlesen beginnt, beendet der Lehrer seine Wartezeit mit dem auffordernden $<<h>m h$ ? $>$ (Z. 29). Hierauf reagiert der Schüler mit der auf Deutsch formulierten Nachfrage soll ich den (...), die durch seine leise Sprechweise und den überlappenden Äußerungsbeginn des Lehrers you nicht zu verstehen ist. Der Lehrer fordert Marius dazu auf, einfach den Steckbrief vorzulesen you just READ what you wrote; wobei es selbst in der Unterrichtssprache Englisch bleibt. Zu erkennen ist erneut eine expressive, beidhändige Gestikulation (Bild 27), die mit einem kurzen Kopfnicken koordiniert ist. Beides unterstreicht neben der Verbalität auch die körperliche Orientierung des Lehrers auf den Schüler.

Im Anschluss wiederholt der Lehrer nunmehr in der Öffentlichkeit die positive Evaluation its very nice, die er bereits zuvor in der Interaktionsdyade auf Deutsch realisiert hatte ( $Z$. 18: das ist schön). Für beide Situationen gilt die projektive Qualität der Evaluation, die als vorgreifender Kredit für die Rezeption des noch nicht vorgelesenen Steckbriefes positive Voraussetzungen schafft.

\section{Bild 28}

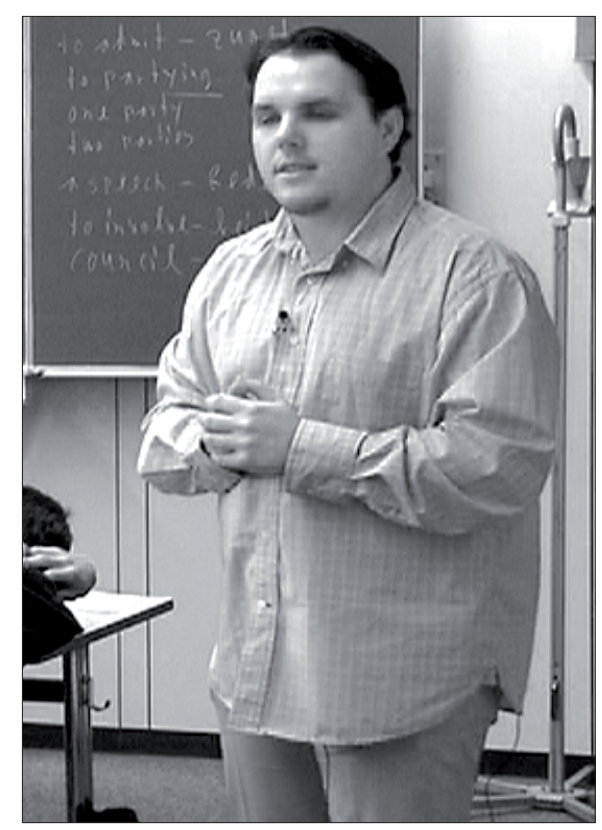

32 WE: its very nice okay,

In körperlicher Hinsicht erkennen wir die Wiederholung eines Aspektes der interpersonellen Koordination (Deppermann/Schmitt 2007) des Lehrers mit dem Schüler: Wie beim Aufruf des Schülers zum Vorlesen des ersten Steckbriefes haben wir auch hier zunächst eine manifeste und expressive Gestikulation, der - zum Zeichen des Wartens und somit quasi als floor-Verdeutlichung für Marius - das Versammeln und die symbolische Rücknahme der eigenen Aktivität (loses Zusammenführen beider Hände, geschlossene Augen) folgen. Das Standbild (Bild 28) verdeutlicht zudem als weiteren Aspekt der körperlichräumlichen Orientierung auf den Schüler, dass sich der Lehrer Marius inzwischen noch etwas mehr angenähert hat. Er ist nun sozusagen im sprichwörtlichen Sinne „,bei ihm“. Er hätte der Präsentation des Steckbriefes auch von seiner angestammten Frontposition zuhören können, was mit dem Vorteil verbunden gewesen wäre, dabei die gesamte Klasse im 
Blick zu haben. Dies ist bei der aktuellen Position des Lehrers in der Nähe von Marius nicht der Fall. Der Lehrer stellt hier also situativ seine Kollektivorientierung auf die gesamte Klasse zugunsten der Individualorientierung auf Marius zurück. ${ }^{12}$

\subsection{Herstellung guter Rezeptionsbedingungen für Marius}

Wie der weitere Fortgang der Interaktion zeigt, kehrt der Lehrer jedoch gleich wieder zu einer expressiven und zunächst ausschließlich auf Marius orientierten bzw. für diesen produzierten Gestikulation zurück (Bild 29, 30).

Bild 29

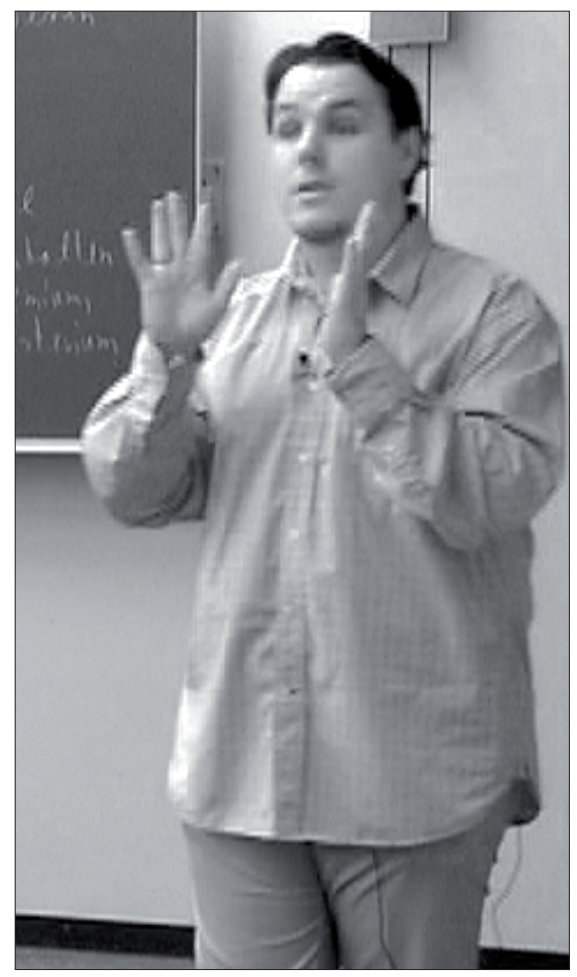

Bild 30

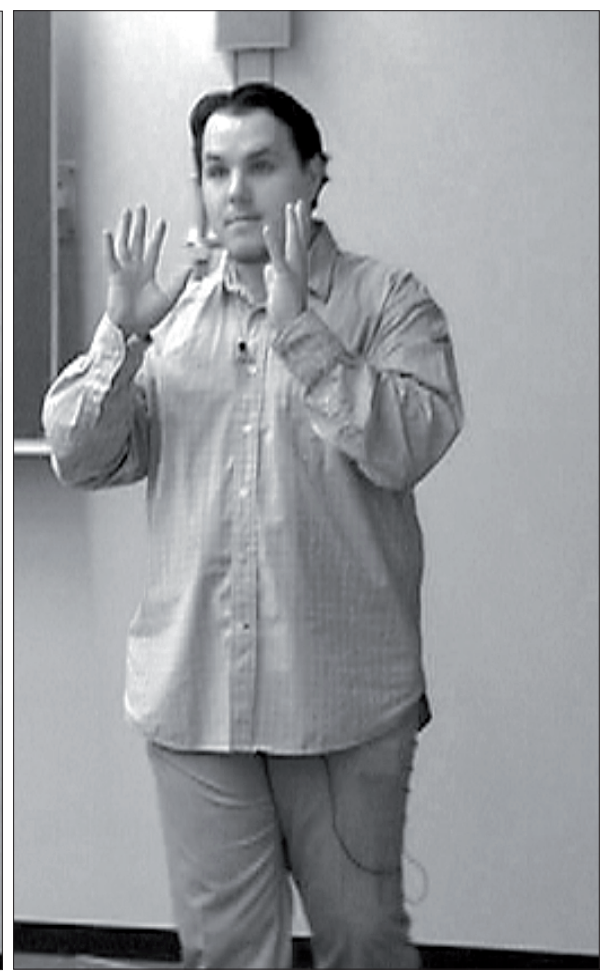

33 WE: and speak LOUDly please okay; (--)

Diese steht im Zusammenhang mit der Herstellung adäquater Rezeptionsbedingungen für die Präsentation des Steckbriefes von Marius. Zur Herstellung adäquater Rezeptionsbedingungen wendet sich der Lehrer zunächst an Marius selbst (Bild 29, 30). Diesen fordert er auf, laut zu sprechen and speak LOUDly please okay; Motiviert wird dieser Hinweis einerseits durch die vorherige leise realisierte Nachfrage des Schülers (Z. 30 soll ich den [(...)]). Hinsichtlich des Explizitheits- und Expansionsgrades, mit denen der Lehrer versucht, adäquate Bedingungen für Marius zu organisieren, spielt vor allem sein Wissen um die „typische Sprechweise dieses Schülers“ eine Rolle.

12 Zur lehrerspezifischen Anforderung und den Verfahren der Bearbeitung der strukturell widerstreitenden Kollektiv- und Individualorientierung siehe Schmitt (im Druck, Kap. II). 
Bild 31

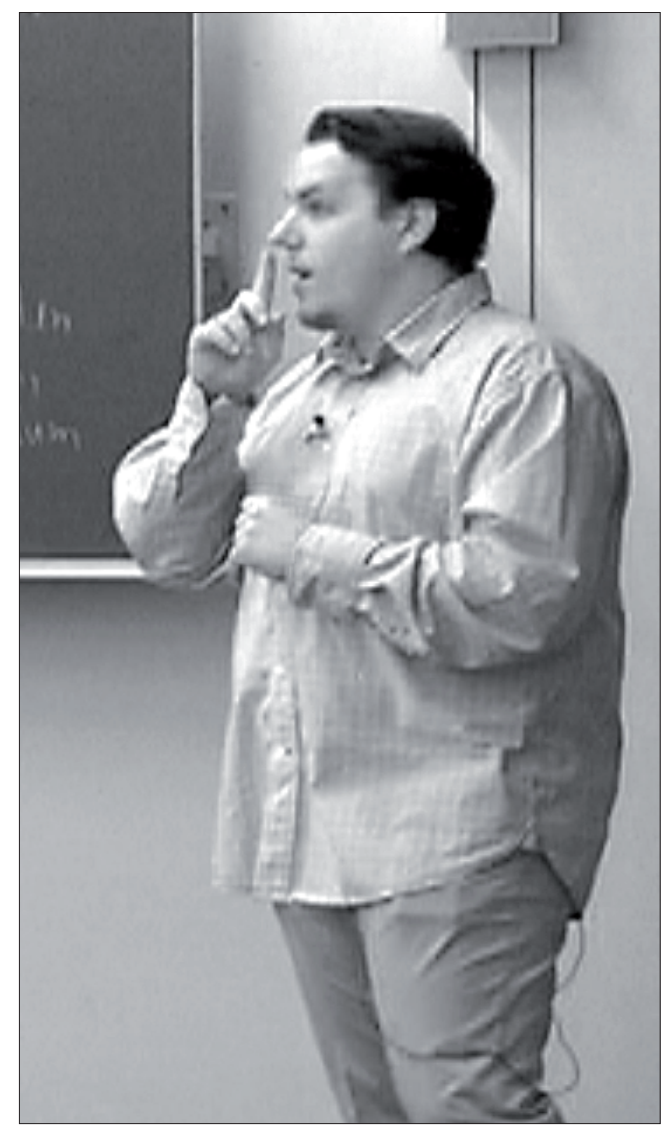

$34 \mathrm{WE}:\langle<f>$ and the others are quiet

35 so we can understand Marius- okay; $>$

Dies wird auch durch seine Hinwendung zur Klasse und die laut realisierte Aufforderung an die übrigen Schüler deutlich, ruhig zu sein, damit Marius verstanden werden kann. Auch hier wird wieder eine explizite, fast zitierende Gestikulation deutlich, als der Lehrer das Ruhig-Sein durch den Zeigefinger vor dem Mund simultan mit der Realisierung von quiet symbolisiert (Bild 31). Auf jeden Fall ist deutlich, dass die beidhändige, auf Marius orientierte Gestikulation des Lehrers ein wesentlicher Teil der speziell auf den Schüler zugeschnittenen Unterstützung ist und der Lehrer seine Adressierung nicht nur verbal, sondern auch gestikulatorisch realisiert.

\subsection{Mithilfe des Lehrers bei der Präsentation von Marius}

Auch bei der nachfolgenden Präsentation des Steckbriefes wird der Lehrer in sehr unterschiedlicher Weise unterstützend tätig.

\subsubsection{Der Lehrer als Sprecher des Schülers}

36 MA: he plays soccer

$37 \mathrm{xX}$ : what

Während der Präsentation des Steckbriefes nimmt der Lehrer eine rezipierende Grundhaltung ein, die sich folgendermaßen charakterisieren lässt: Er steht mit vor der Brust verschränkten Händen auf Marius blickend eine Tischreihe von dem Schüler entfernt. Diese 
Haltung modifiziert er nur in Reaktion auf einzelne Aspekte der Steckbrief-Präsentation. In bestimmten Momenten dreht er sich einerseits nach rechts, um in die Klasse zu blicken, oder er löst seine lose gefalteten Hände, um auf Marius orientiert und an diesen gerichtet, zu gestikulieren. Die erste Hinwendung zur Klasse erfolgt in Reaktion auf die Nachfrage eines nicht identifizierbaren Sprechers what? (Z. 37).

Bild 32
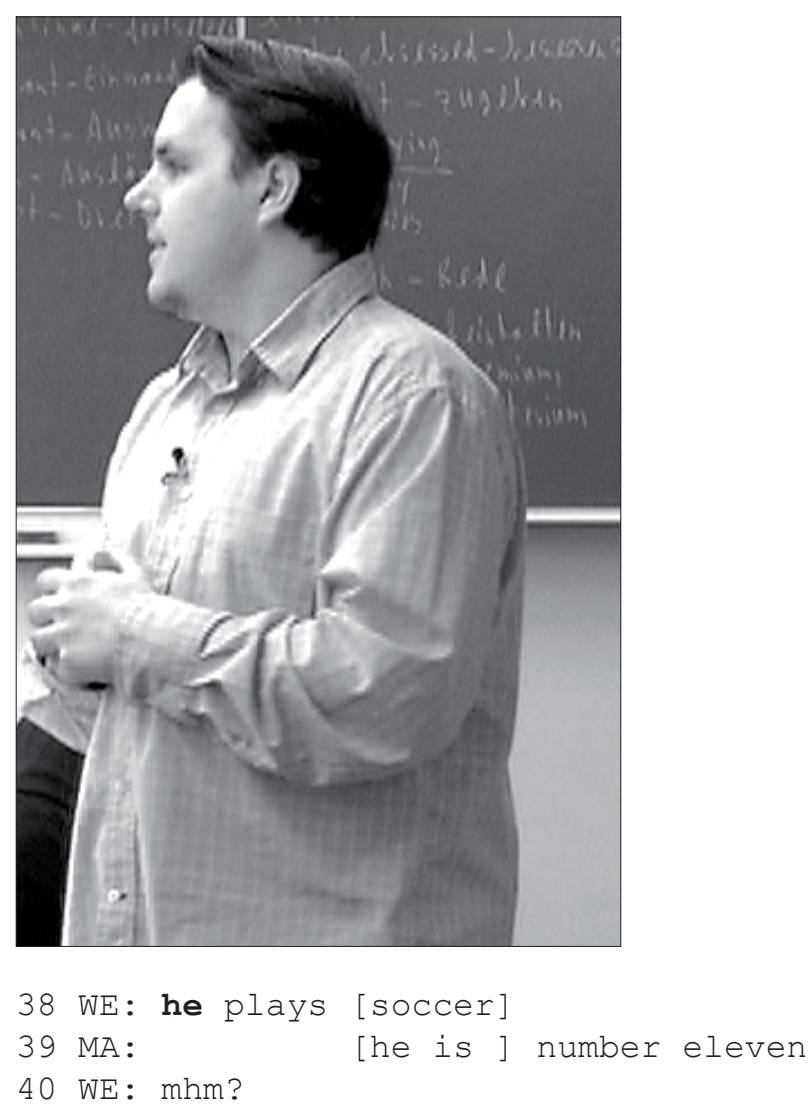

Statt Marius die Möglichkeit zu geben, in Reaktion auf diese Nachfrage den fraglichen Teil noch einmal vorzulesen, agiert der Lehrer für den Schüler. Er blickt in die Klasse (Bild 32) und macht sich zum Sprecher des Schülers, indem er mit lauter Stimme den ersten Teil des Steckbriefes he plays soccer wiederholt. Marius hatte trotz der unmittelbar vorangegangenen Aufforderung des Lehrers, laut zu sprechen, diesen Teil mit leiser Stimme vorgetragen und dadurch die Nachfrage what (Z. 37) motiviert. Dadurch, dass der Lehrer für Marius spricht, überlappt er den Anfang der zweiten Information des Steckbriefes [he is]. Er schafft dadurch selbst und unabsichtlich erschwerte Rezeptionsbedingungen. Der Lehrer ratifiziert dann die zweite Steckbriefinformation mit dem zustimmenden mhm? (Z. 40).

\subsubsection{Implizite und aspektualisierte Korrektur}

Der Lehrer folgt dann aus seiner Rezeptionshaltung heraus der weiteren Präsentation des Steckbriefes. Er nutzt eine Pause beim Vorlesen dazu, einen Fehler des Schülers zu korrigieren. Er substituiert die deutschsprachige Abkürzung wm (Z. 42) durch die korrekte englische Bezeichnung worldcup (Z. 43). 


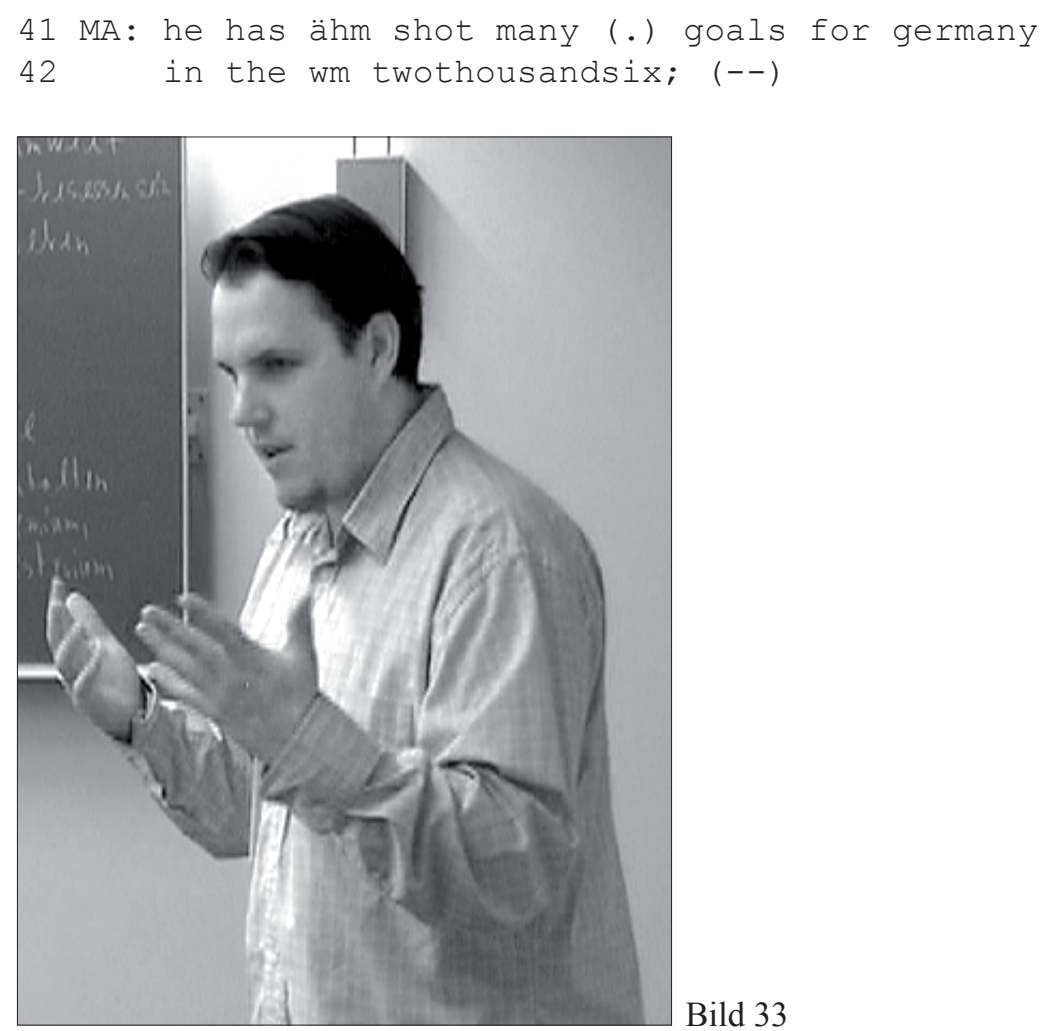

43 WE: in the worldcup twothousandandsix (.) yes;

Hinsichtlich der Korrektur sind zwei Punkte interessant. Zum einen verzichtet der Lehrer weitgehend auf Kontrastivität zur Verdeutlichung von richtig und falsch. Zum anderen korrigiert er nur einen von zwei Fehlern. Er übergeht nämlich den falschen Tempusgebrauch he has shoot statt he shot oder he marked (many goals). Deutlich bei dieser Korrektur ist wieder ihre Indirektheit und Implizitheit, die wir bereits bei der Korrektur in der Dyade gesehen haben. Zum anderen gestikuliert der Lehrer erneut beidhändig und expressiv in Richtung des Schülers, den er zudem anschaut (Bild 33).

\subsubsection{Der Lehrer als Co-Autor und Mitwisser}

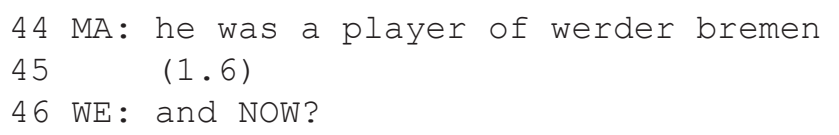

Der Lehrer, der den Steckbrief zuvor in der dyadischen Konstellation gelesen hatte, merkt, dass Marius den Text nicht vollständig vorgelesen und das Blatt zu früh bereits wieder auf dem Tisch abgelegt hat. Daher fordert er ihn mit der Frage and NOW? (Z. 46 mit kontrastivem Akzent auf NOW?) zum Nachliefern der letzten Information über den Spieler auf. Während er darauf wartet, dass Marius das Blatt wieder aufnimmt, um den Text vollständig vorzulesen, hat er seinen Kopf leicht angehoben und lächelt dem Schüler zu (Bild 34). 


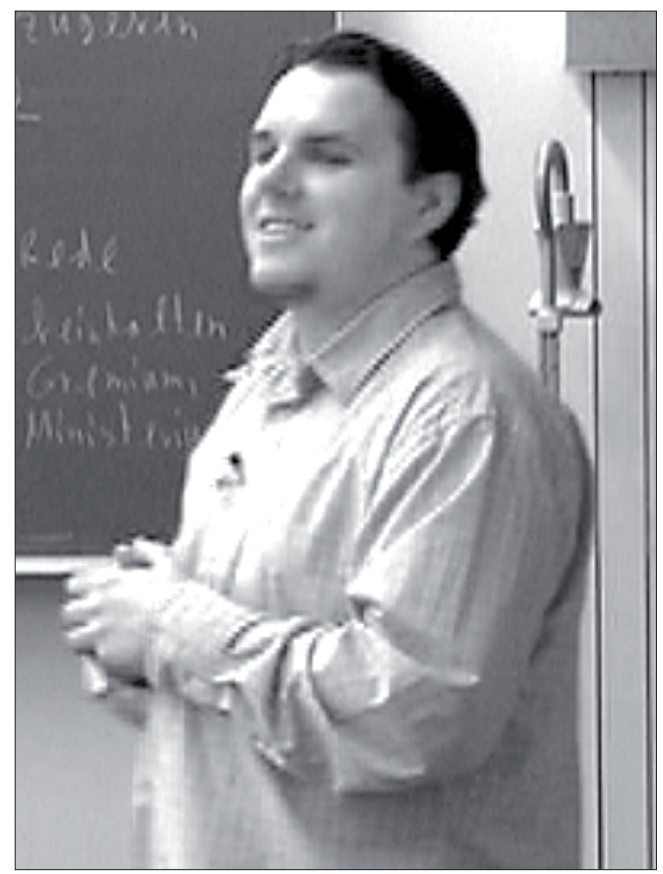

$(2.1)$

48 MA: and now he plays for bayern münchen

49 WE: mhm? Erol;

\subsubsection{Aufruf zum Raten der Person und Ratifikationseinholung bei Marius}

Nachdem Marius mit and now he plays for bayern münchen die fehlende Information nachgeliefert hat, wendet sich der Lehrer wieder der Klasse zu und ruft einen Schüler, der sich bereits seit einiger Zeit gemeldet hat, zur Nennung des Spielers auf (Z. 49 Erol?).

50 ER: äh miroslav klose

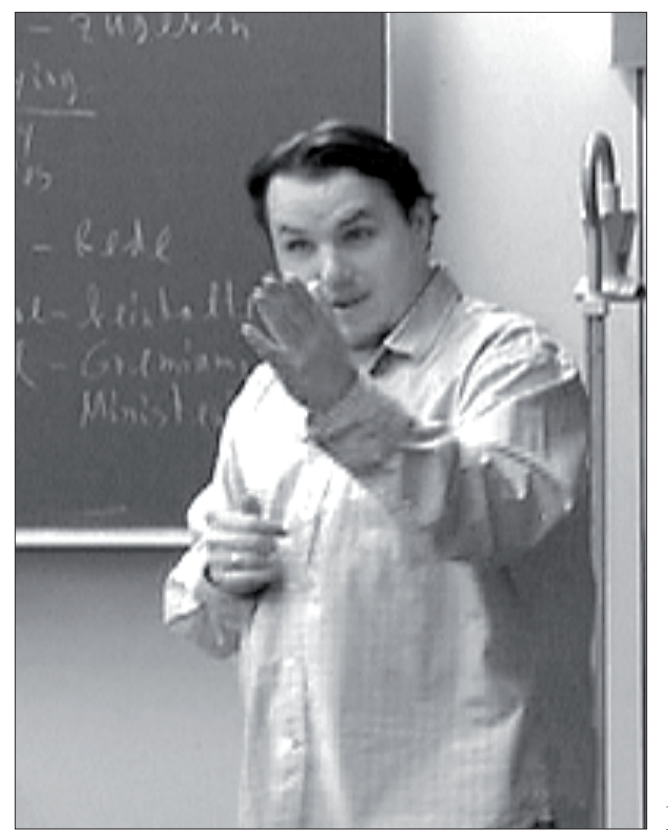

51 WE: is that correct? (-)

52 MA: $<<$ p $>$ nickt $>$ 
Der Lehrer liefert die Ratifikation des genannten Namens nicht selbst, sondern wendet sich an Marius und lässt sich von diesem die Richtigkeit des Namens bestätigen. Auf diese Weise symbolisiert er die Zuständigkeit des Schülers für die Ratifikation. Auch hier unterstreicht der Lehrer seine Orientierung auf den Schüler mit einer manifesten Zeigegestikulation (Bild 35).

\subsection{Analyse-Resümee (2)}

\section{Markierung von Hilfsbedürftigkeit durch den Schüler}

Der Schüler dokumentiert seinen Unterstützungsbedarf in der zweiten Phase vor allem durch die Verzögerung des Vorlesens, die explizite Nachfrage zum Vorlesestart und durch eine sehr leise vorgetragene Präsentation des Steckbriefes und eine Orientierung auf schnellen Abschluss. Letztere führt dazu, dass er vergisst, einen Teil des Steckbriefes vorzulesen, und sehr früh sein Blatt mit der Textseite nach unten ablegt.

\section{Unterstützungen durch den Lehrer}

Zu den Unterstützungsaktivitäten des Lehrers gehören in der zweiten Phase vor allem die explizite, sehr freundliche Aufforderung zum Vorlesen, die vorgezogene Positivevaluation im Zusammenhang mit der Wiederholung der Vorleseaufforderung, die explizite Herstellung adäquater Rezeptionsbedingungen für die Präsentation des Schülers mit doppelter Adressierung (Marius und die restlichen Schüler), die Übernahme der Sprecherrolle für den Schüler und die laute Wiederholung der von Marius leise gelieferten Informationen, das Agieren als Mitwisser und die damit zusammenhängende Elizitierung der letzten wichtigen Information des Steckbriefes, die Marius nicht (mehr) vorgelesen hat, sowie die mit der Ratifikationsnachfrage (is that correct?) verbundene Zuschreibung von Zuständigkeit und Expertenstatus.

Das unterstützende Verhalten des Lehrers wird durch eine paradoxale Implikation geprägt. Je manifester und kontinuierlicher er den Schüler unterstützt, desto deutlicher macht er dessen Hilfsbedürftigkeit, da der Schüler nicht in der Lage ist, sich in der Rolle des Präsentierenden selbst ohne Hilfe zu vertreten: Er spricht $\mathrm{zu}$ leise und vergisst relevante Informationen. Ergänzt wird seine Unterstützung des Schülers durch eine Nähe-DistanzRegulierung und eine expressive schülerbezogene Gestikulation, die kontinuierlich die Bezogenheit des Lehrers auf den Schüler verkörpern. Zusätzlich evaluiert der Lehrer jede neue Information des Steckbriefes mit positiven verbalen Rückmeldern und durch Kopfnicken. Er inszeniert sich dadurch als aufmerksamer Rezipient und macht aus der kurzen Präsentation ein interaktives Ereignis mit eigener aktiver Beteiligung.

\section{Unterstützen als professionelles Handeln}

„Unterstützen im Gespräch“ wurde als gesprächsrhetorisches Verfahren ${ }^{13}$ zur Bearbeitung lokaler interaktiver Anforderungen in Schmitt (1998) untersucht. Unterstützen wurde dabei einerseits in seiner interaktionsstrukturellen Spezifik rekonstruiert und andererseits hinsichtlich seiner sozialen Implikationen analysiert. Im Unterschied zur hier analysierten Unterstützungsinteraktion im Unterricht zeichnete sich die empirische Grundlage der damaligen Untersuchung durch eine grundsätzliche Egalität der interaktiven Beteiligungs-

13 Zum gesprächsrhetorischen Ansatz siehe Kallmeyer (1996) und als methodische Exemplifizierung Kallmeyer/Schmitt (1996). 
weise aus. Es gab zwischen Unterstützer und Unterstütztem keinerlei hierarchie- oder statusbezogenes Gefälle oder eine rollenspezifische Konstellation. Für diesen egalitären Beteiligungskontext wurde auf der Grundlage einer „Präferenz für kommunikative Selbstvertretung“ Unterstützen als strukturell ambivalenter Zusammenhang deutlich. Durch die unterstützenden Aktivitäten eines Beteiligten, die auf einen interaktiven Vorfall und die Signalisierung von Unterstützungsbedarf eines anderen Beteiligten reagieren, wird die lokale Unfähigkeit zur interaktiven Selbstvertretung offensichtlich und der interaktive Status des unterstützten Beteiligten als hilfsbedürftig manifest. Dies ist völlig unabhängig von den Intentionen des Unterstützers der Fall und liegt in der ambivalenten Struktur des gesprächsrhetorischen Verfahrens selbst.

Welche Implikationen sind mit unterstützenden Aktivitäten verbunden, in denen sich die Unterstützungsinteraktion als konstitutiver und erwartbarer Teil des professionellen Handelns des Lehrers vollzieht? Beantwortet man diese Frage auf der Grundlage der zurückliegenden Analyse, wird folgender Unterschied hinsichtlich a) der sozialen Implikationen und b) bezogen auf die eingesetzten multimodalen Ressourcen bei der Unterstützung des Lehrers deutlich.

Ad a) Die sozialen Implikationen, die mit der Präferenz zur interaktiven Selbstvertretung zusammenhängen und sich als Dokumentation der Hilfsbedürftigkeit des Unterstützten niederschlagen, realisieren sich zunächst nur in der Öffentlichkeit der Klasse, nicht jedoch in der dyadischen Konstellation. Im Vergleich mit nichtpädagogischen, egalitären Kontexten sind es vor allem situations- und organisationsstrukturelle Aspekte, welche die Relevanz der Präferenz für interaktive Selbstvertretung als primäre Handlungsorientierung relativieren. Im gemeinsamen Wissen der am Unterricht Beteiligten ist verankert, dass es Bestandteil des pädagogischen Auftrags des Lehrers ist, nicht nur in ganz allgemeiner Weise Schüler beim Lernen und bei der Wissensvermittlung zu unterstützen. Er hat darüber hinaus die Aufgabe, möglichst alle Schüler aktiv am Unterricht zu beteiligen und unterliegt in besonderer Weise einer Fürsorge- und Förderpflicht schwächerer und interaktiv zurückhaltender Schüler. Die Organisation von Beteiligungs- und Darstellungsgelegenheiten gerade schwächerer Schüler gehört also zum Unterrichtsalltag. Im vorliegenden Fall haben wir es darüber hinaus mit einer Unterstützung zu tun, die aufgrund ihrer Intensität und Dauer eine eigenständige Bedeutung erhält.

Gleichwohl - und darin sind „Unterstützen im (alltagsweltlichen) Gespräch“ und „Unterstützen als professionelles Handeln" strukturell vergleichbar - steckt auch der Lehrer in einem nicht lösbaren Dilemma: Auch er macht durch die Spezifik seiner Unterstützung nicht nur die Unterstützungsbedürftigkeit des Schülers, sondern im weiteren Sinne auch dessen Status im Klassenverbund aus seiner Perspektive deutlich. Auch „Unterstützen als professionelles Handeln“ bezieht sich ausschließlich auf die Organisation der lokalen interaktiven Beteiligung des Unterstützten. Unterstützen ist auch im professionellen Handlungszusammenhang ein gesprächsrhetorisches Verfahren und unterliegt dessen strukturellen Implikationen. Unterstützen ist in diesem Sinne nicht geeignet, statusverändernde Hilfestellungen zu geben. Der unterstützte Schüler wird auch nach der Unterstützung den untergeordneten und peripheren Status im Klassenverband innehaben, der den Lehrer zu genau der komplexen multimodalen Gesamtgestalt seiner Unterstützung veranlasst hat. 
Aber es gelingt dem Lehrer durch sein unterstützendes Verhalten immerhin, dem Schüler lokal eine Darstellungsgelegenheit zu organisieren, die er von sich aus nicht ergriffen hätte. Der Lehrer rückt - bei aller strukturellen Ambivalenz seiner Unterstützung - den unterstützten Schüler für einen Moment in den Wahrnehmungsfokus der Klassenöffentlichkeit (einige Schüler drehen sich zu Marius um, sie hören ihm zu, erraten die von ihm beschriebene Person etc.) und macht ihn dadurch als inhaltlich kompetent und sozial existent für alle sichtbar.

Ad b) Hinsichtlich der eingesetzten modalitätsspezifischen Ressourcen des Lehrers wird ersichtlich, dass sie in der Dyade primär auf die Herstellung und Symbolisierung von Nähe und Ko-Orientierung ausgerichtet sind. Der Lehrer steht so nahe bei dem Schüler, dass sich deren Körper und situativ die Hände fast berühren. In der Öffentlichkeit der Klasse zielen die eingesetzten Ressourcen primär auf die Verdeutlichung der Orientierung des Lehrers auf den Schüler aus einer Mitteldistanz heraus: Er steht erkennbar weiter von ihm weg und gestikuliert expressiv und adressiert in die Richtung des Schülers. Die Mitteldistanz ist hier motiviert durch die Doppelorientierung des Lehrers, nämlich neben seiner Individualorientierung auf Marius auch der Kollektivorientierung auf die Klasse insgesamt zu folgen.

Neben der verbal geleisteten Hilfestellung symbolisiert der Lehrer in dem analysierten Ausschnitt kontinuierlich seine Haltung und Einstellung dem Schüler gegenüber. Unterstützen als multimodales Gesamtpaket hat im professionellen Handlungsfeld „Unterricht“ eher die Struktur einer dauerhaften Symbolisierung als die einer lokalen, formal-strukturellen Bearbeitung einer identifizierbaren und in der Interaktionsstruktur klar lokal identifizierbaren Anforderung. So ist es nicht verwunderlich, dass sich Spuren der analysierten Unterstützung auch noch bei der Rückführung zum Unterricht nach dem Intermezzo „Ausländer in der deutschen Nationalmannschaft“ (siehe Fallanalyse 3) finden lassen. Unterstützen als konstitutive Anforderung professionellen Handelns im Unterricht ist über seine Oberflächenstruktur, bei der es um konkrete, thematisch-praktische Belange geht, immer tief gegründet in der kontinuierlichen Anforderung des Klassenmanagements und der situativen Balancierung der Individual- und Kollektivorientierung der Lehrer/innen.

\section{Literatur}

Bönsch, Manfred (1995): Differenzierung in Schule und Unterricht. Ansprüche-Formen-Strategien. Berlin: Oldenburg bsv.

Deppermann, Arnulf/Schmitt, Reinhold (2007): Koordination. Zur Begründung eines neuen Forschungsgegenstandes. In: Schmitt, Reinhold (Hg.): Koordination. Analysen zur multimodalen Interaktion. Tübingen: Narr. S. 15-54.

Deppermann, Arnulf/Schmitt, Reinhold (2008): Verstehensdokumentationen: Zur Phänomenologie von Verstehen in der Interaktion. In: Deutsche Sprache 36, S. 220-245.

Garfinkel, Harold (1967): Studies in ethnomethodology. Englewood Cliffs, New Jersey.

Goffman, Erving (1983): The interaction order. In: American Sociological Review 48, S. 1-17.

Goodwin, Charles (2007): Environmentally coupled gestures. In: Duncan, Susan D./Cassell, Justine/Levy, Elena T. (Hg.): Gesture and the Dynamic Dimension of Language. Amsterdam/Philadelphia: Benjamins. S. 195-212. 
Hall, Edward T. (1966): The hidden dimension. New York: Random House.

Hausendorf, Heiko/Mondada, Lorenza/Schmitt, Reinhold (Hg.) (2012): Raum als interaktive Ressource. Tübingen: Narr.

Hausendorf, Heiko/Mondada, Lorenza/Schmitt, Reinhold (2012): Raum als interaktive Ressource. Eine Explikation. In: Hausendorf, Heiko/Mondada, Lorenza/Schmitt, Reinhold (Hg.): Raum als interaktive Ressource. Tübingen: Narr. S. 7-36.

Hausendorf, Heiko/Schmitt, Reinhold (2010): Opening up Openings. Zur multimodalen Konstitution der Eröffnungsphase eines Gottesdienstes. In: Mondada, Lorenza/Schmitt, Reinhold (Hg.): Situationseröffnungen. Zur multimodalen Herstellung fokussierter Interaktion. Tübingen: Narr. S. 53-101.

Heidtmann, Daniela/Schmitt, Reinhold (2011): Interaktives Diktieren. Komplexe Anforderungen im Epochen-Unterricht. In: Schmitt, Reinhold (Hg.): Unterricht ist Interaktion! Analysen zur De-facto-Didaktik. Mannheim: IDS. S. 179-224. (= amades 41).

Helmke, Andreas/Schrader, Friedrich-Wilhelm (2009): Qualitätsmerkmale „guten Unterrichts“. In: Hellekamps, Stephanie/Plöger, Wilfried/Wittenbruch, Wilhelm (Hg.): Handbuch für Erziehungswissenschaft, Band II/1: Schule. Paderborn: Schöningh. S. 701-712.

Kallmeyer, Werner (1996): Einleitung: Was ist Gesprächsrhetorik? In: Kallmeyer, Werner (Hg.): Gesprächsrhetorik. Rhetorische Verfahren im Gesprächsprozeß. Tübingen: Narr. S. 7-18.

Kallmeyer, Werner/Schmitt, Reinhold (1996): Forcieren oder: Die verschärfte Gangart. Zur Analyse von Kooperationsformen im Gespräch. In: Kallmeyer, Werner (Hg.): Gesprächsrhetorik. Rhetorische Verfahren im Gesprächsprozeß. Tübingen: Narr. S. 20-118.

Kendon, Adam (1990): Conducting interaction. Patterns of behavior in focused encounters. Cambridge: Cambridge University Press.

Kuty, Margitta (2009): „Innere Differenzierung“ und „Individualisierung“. In: Didaktisches Lexikon. (Internet: www.oldenbourg.de/osv/zeitschriften/fsu/didaktischeslexikon/pfu20090362.pdf).

Lee, Yo-An (2007): Third turn position in teacher talk: Contigency and the work of teaching. In: Journal of Pragmatics 39, S. 1204-1230.

Lemke, Jay L. (1990): Talking science: Language, learning and value. New York: Ablex.

Malone, Martin J. (1997): Worlds of talk. The presentation of self in everyday conversation. Cambridge: Polity Press.

Mannheim, Karl (1952): On the interpretation of 'Weltanschauung'. In: Mannheim, Karl: Essays in the sociology of knowledge. Ed. by Paul Kecskemeti. London: Routledge. S. 33-83.

Markee, Numa (2004): The organization of off-talk talk in second language classrooms. In: Richards, Keith/ Seedhouse, Paul (Hg.): Applying conversation analysis. Basingstoke: Palgrave Macmillan. S. 197-213.

McHoul, Alexander (1978): The organization of turns at formal talk in the classroom. In: Language in Society 7, S. 183-213.

Mehan, Hugh (1979): Learning lesson. Cambridge: Harvard University Press.

Meyer, Hilbert (2004): Was ist guter Unterricht? Berlin: Cornelsen.

Mondada, Lorenza (2004): Temporalité, séquentialité et multimodalité au fondement de l'organisation de l'interaction: Le pointage comme pratique de prise du tour. In: Cahiers de Linguistique Française 26, S. 169-192.

Mondada, Lorenza (2006): Participants' online analysis and multimodal practices: projecting the end of the turn and the closing of the sequence. In: Discourse Studies 8, S. 117-129.

Mondada, Lorenza (2007a): Multimodal resources for turn-taking: Pointing and the emergence of possible next speakers. Discourse Studies 9/2, S. 195-226. 
Mondada, Lorenza (2007b): Deixis spatiale, gestes de pointage et formes de coordination de l'action. In: Barbéris, Jean-Marie/Manes-Gallo, Marie-Caterina (Hg.): Parcours dans la ville. Les descriptions d'itinéraires piétons. Paris: L'Harmattan. S. 261-285.

Mondada, Lorenza/Schmitt, Reinhold (Hg.) (2010): Situationseröffnungen: Zur multimodalen Herstellung fokussierter Interaktion. Tübingen: Narr.

Müller, Cornelia/Bohle, Ulrike (2007): Das Fundament fokussierter Interaktion Zur Vorbereitung und Herstellung von Interaktionsräumen durch körperliche Koordination. In: Schmitt, Reinhold (Hg.): Koordination. Analysen zur multimodalen Interaktion. Tübingen: Narr. S. 129-165.

Nassaji, Hossein/Wells, Gordon (2000): What's the use of "Triadic dialogue"? An investigation of teacherstudent interaction. In: Applied Linguistics 21, S. 376-406.

Sacks, Harvey (1992): Lectures on Conversation. 2 Bände. Oxford: Blackwell.

Sacks, Harvey/Schegloff, Emanuel A./Jefferson, Gail (1974): A simplest systematics for the organization of turn-taking in conversation. In: Language 50/4, S. 696-735.

Schmitt, Reinhold (1997): Unterstützen im Gespräch. Zur Analyse manifester Kooperationsverfahren. In: Zeitschrift für Sprachwissenschaft 16/1-2, S. 52-82.

Schmitt, Reinhold (2004): Die Gesprächspause: „Verbale Auszeiten“ aus mulimodaler Perspektive. In: Deutsche Sprache 32, S. 56-84.

Schmitt, Reinhold (2006): Videoaufzeichnungen als Grundlage für Interaktionsanalysen. In: Dickgießer, Sylvia/Reitemeier, Ulrich/Schütte, Wilfried (Hg.): „Symbolische Interaktionen“ (Sonderheft Deutsche Sprache 34, 1-2). Berlin, S. 18-31.

Schmitt, Reinhold (Hg.) (2007a): Koordination. Analysen zur multimodalen Interaktion. (= Studien zur Deutschen Sprache 38). Tübingen: Narr.

Schmitt, Reinhold (2007b): Von der Konversationsanalyse zur Analyse multimodaler Interaktion. In: Kämper, Heidrun/Eichinger, Ludwig M. (Hg.): Sprachperspektiven. Germanistische Linguistik und das Institut für Deutsche Sprache. Tübingen: Narr. S. 395-417. (= Studien zur Deutschen Sprache 40).

Schmitt, Reinhold (2007c): Theoretische und methodische Implikationen der Analyse multimodaler Interaktion. In: Holly, Werner/Paul, Ingwer (Hg.): Medialität und Sprache. Bielefeld: Aisthesis. S. 26-53. (= Mitteilungen des Deutschen Germanistenverbandes 54/1).

Schmitt, Reinhold (2010): Verfahren der Verstehensdokumentation am Filmset: Antizipatorische Initiativen und probeweise Konzeptrealisierung. In: Deppermann, Arnulf et al. (Hg.): Verstehen in professionellen Handlungsfeldern. Tübingen: Narr. S. 210-362.

Schmitt, Reinhold (2011a): Unterricht ist Interaktion. Eine Rahmung. In: Schmitt, Reinhold (Hg.): Unterricht ist Interaktion! Analysen zur De-facto-Didaktik. Mannheim:IDS. S. 7-30. (= amades 41).

Schmitt, Reinhold (2011b): Didaktik aus interaktionistischer Sicht. Das Konzept „De-facto-Didaktik“. In: Schmitt, Reinhold (Hg.): Unterricht ist Interaktion! Analysen zur De-facto-Didaktik. Mannheim: IDS. S. 225-238. (= amades 41).

Schmitt, Reinhold (2012 a): Gehen als situierte Praktik: „Gemeinsam gehen“ und ,hinter jemandem herlaufen“. In: Gesprächsforschung Online 13, S. 1-44. (www.gespraechsforschung-ozs.de).

Schmitt, Reinhold (2012b): Räumliche Grundlagen interaktiver Beteiligung. Das Konzept „Interaktionsensemble“. In: Hausendorf, Heiko/Mondada, Lorenza/Schmitt, Reinhold (Hg.): Raum als interaktive Ressource. Tübingen: Narr. S. 37-87.

Schmitt, Reinhold (im Druck): Körperlich-räumliche Aspekte der Interaktion. Tübingen: Narr.

Schmitt, Reinhold/Deppermann, Arnulf (2007): Monitoring und Koordination als Voraussetzungen der multimodalen Konstitution von Interaktionsräumen. In: Schmitt, Reinhold (Hg.): Koordination. Analysen zur multimodalen Interaktion. Tübingen: Narr. S. 95-128. 
Schmitt, Reinhold/Deppermann, Arnulf (2009): „,damit sie mich verstehen“: Genese, Verfahren und recipient design einer narrativen Performance. In: Habscheid, Stephan et al. (Hg.): Theatralität des sprachlichen Handelns. Eine Metaphorik zwischen Linguistik und Kulturwissenschaften. München: Fink. S. 79-112.

Schmitt, Reinhold/Deppermann, Arnulf (2010): Die Transition von Interaktionsräumen als Situationseröffnung. In: Mondada, Lorenza/Schmitt, Reinhold (Hg.): Situationseröffnungen: Zur multimodalen Herstellung fokussierter Interaktion. Tübingen: Narr. S. 335-386.

Sinclair, John/Coulthard, Malcom (1975): Towards an analysis of spoken discourse: The English used by teachers and pupils. Oxford: Oxford University Press.

van Lier, Leo (1988): The classroom and the language learner. London: Longman.

Dr. Reinhold Schmitt

Institut für Deutsche Sprache

Postfach 101621

D-68016 Mannheim

E-Mail: reinhold.schmitt@ids-mannheim.de 